\title{
Nanobeams and AFM Subject to Piezoelectric and Surface Scale Effects
}

\author{
Julio Claeyssen $\left(\mathbb{D},{ }^{1,2}\right.$ Rosemaira Copetti, ${ }^{1}$ Leticia Tonetto, ${ }^{3}$ and Joao Carvalho ${ }^{2}$ \\ ${ }^{1}$ PGMAT, Departamento de Matemática, CCNE, Universidade Federal de Santa Maria, Av. Roraima 1000, 97105-900, \\ Santa Maria, RS, Brazil \\ ${ }^{2}$ PPGMAp-IME, Universidade Federal do Rio Grande do Sul, Av. Bento Gonçalves, 9500, 91509-900, Porto Alegre, RS, Brazil \\ ${ }^{3}$ Centro de Engenharias, CENG, Universidade Federal de Pelotas, Almirante Barroso, 1734, Pelotas, RS, 96010-280, Brazil
}

Correspondence should be addressed to Julio Claeyssen; jcrclaeyssen@yahoo.com

Received 22 July 2018; Accepted 16 September 2018; Published 18 October 2018

Academic Editor: Phuc Phung-Van

Copyright (C) 2018 Julio Claeyssen et al. This is an open access article distributed under the Creative Commons Attribution License, which permits unrestricted use, distribution, and reproduction in any medium, provided the original work is properly cited.

\begin{abstract}
Vibration dynamics of elastic beams that are used in nanotechnology, such as atomic force microscope modeling and carbon nanotubes, are considered in terms of a fundamental response within a matrix framework. The modeling equations with piezoelectric and surface scale effects are written as a matrix differential equation subject to tip-sample general boundary conditions and to compatibility conditions for the case of multispan beams. We considered a quadratic and a cubic eigenvalue problem related to the inclusion of smart materials and surface effects. Simulations were performed for a two stepped beam with a piezoelectric patch subject to pulse forcing terms. Results with Timoshenko models that include surface effects are presented for micro- and nanoscale. It was observed that the effects are significant just in nanoscale. We also simulate the frequency effects of a doublespan beam in which one segment includes rotatory inertia and shear deformation and the other one neglects both phenomena. The proposed analytical methodology can be useful in the design of micro- and nanoresonator structures that involve deformable flexural models for detecting and imaging of physical and biochemical quantities.
\end{abstract}

\section{Introduction}

Atomic force microscopy (AFM) is a scanning probe microscopy (SPM) technique to obtain images of surface topography at the atomic scale, in a noninvasive manner, from a wide variety of samples on a scale from angstroms to 100 microns [1]. A typical AFM consists of a microcantilever with a sharp tip, a sample positioning system, a detection system, and a control system. The associated length scales are sufficiently small to call the applicability of classical continuum models into question [2]. In this work, we seek to develop a vibration dynamics framework for beams that include smart materials and subject to surface effects. This framework is also considered in the case of a two-span beam in which the first segment is governed by the Timoshenko model and the second segment is an Euler-Bernoulli beam model [3].

Recently, new generations of active microcantilevers have included piezoelectric materials locally attached at the microbeam with the role of sensors and/or actuators linear and nonlinear [4-9], among others. This has led to the study of multispan beams for AFM. The inclusion of smart materials layers will modify material properties between neighboring segments, producing discontinuities and fulfilling compatibility conditions for the continuity of the displacement and rotation of the beam and for the equilibrium of bending moment and shear at the discontinuity points.

Surface effects often play a significant role in the physical properties of micro- and nanosized materials and structures. Since the atoms within a very thin layer near surfaces experience a different local environment form that is experienced by atoms in bulk, the physical properties and mechanical response of surfaces will be distinct from those of bulk materials. Contrary to macroscopic structures, surface effects can strongly influence the stress and deformation properties of nanodevices. This latter is due to the increasing ratio between surface/interface area and volume. For instance, for 
a bulk deformation energy $U_{b} \approx E d^{3}$ ( $E$ elastic stiffness) and $U_{s} \approx \gamma d^{2}$ ( $\gamma$ surface energy per area), we shall have $U_{s}>U_{b}$ with distance $d<\gamma / E$. In [10], it has been shown that with a modified Timoshenko beam model the dynamics of nanoscaled tubes with surface effects deviate considerably from results obtained with classic theories.

In some models, considering effective properties $(D)$ of nanosized beams when compared with standard continuum mechanics $\left(D_{c}\right)$, the relative error $\left(D-D_{c}\right) / D_{c}$ scale is found as $\alpha S / E d$, where $\alpha$ is a geometrical constant depending upon the nanobeam considered, $S$ is a surface elastic constant, $E$ is a bulk elastic modulus, and $d$ is a length defining the size of the structural element [11]. Here we shall consider free vibration of a nanoscale continuum beam models which incorporates surface energy [12-14]. The search for time exponential solutions leads to a quadratic and cubic eigenvalue problems associated with a second-order modal differential system and a singular third-order differential system, respectively.

Simulations have been performed for triple span beams with and without a patch in the first segment and with loads in the first and last segment with tip-sample interaction. These loads included a uniform pulse load and its second derivative associated with the moment due to a piezoelectric patch. It is observed how the inclusion of piezoelectric materials absorbs vibrations when compared with classical multispan beams. Also, the robustness of the impulse response method with varying parameters and its influence in the behavior of the responses are observed.

The size dependence effects in Timoshenko microbeams with surface effects (TMB) have been simulated for the nondimensional natural frequency and compared with those of the classical Timoshenko beam model (TB). It has been observed that for beam length on the order of nanometer to microns, the difference between natural frequencies is apparent, and by increasing the length of the microbeam, the results tend to Timoshenko classical theory; that is, the surface effects are significant only in nanoscale. This same behavior was observed in [14] for a microbeam simply supported.

This work involves a methodology that may be adequately adapted to study recent advances in micro-/nanosized structures that are intensively used to design advanced micro-/nanosensors and molecular transportation systems devices for various engineering and medical applications. These sensors due to their ultrahigh resonant frequencies are important in sensitive sensing, molecular transportation, molecular separation, high-frequency signal processing, and biological imaging. Some deposited processes in the formation of thin-film composite membranes can be similar to types of multispan vibrating beams. However, the complex configuration of materials used in real world devices requires the study of material properties stemming from their atomically thin layered structures. Chemical deposited processes in the manufacturing of thin films on semiconductors can involve low temperatures with silicon rich nitride. Conventional structural analysis methods assume ideal structures (free of irregularities) but material and/or geometrical variations in a structure may result in drastic changes in its dynamical behaviors. Diffusion- and reaction-controlled interfacial polymerization is an important and practical topic that is beyond our scope [15-19].

This paper is organized as follows. In Section 2, we consider the Euler-Bernoulli multispan beam model, their compatibility conditions, and the boundary conditions in a matrix form. In Section 3, the dynamic response of the matrix Euler-Bernoulli multispan beam model subject to tipsample interactions and external forcing is given in terms of the distributed matrix impulse response. The case of a microcantilever with a piezoelectric layer is discussed in Section 4. In Section 5 are presented the results of a doublespan beam, obtained by the expansion of the first segment of the triple piezoelectric beam. In Sections 6 and 7 is considered the eigenanalysis of Timoshenko beam models with surface effects and their comparison with the Timoshenko classical model, for micro- and nanoscale. In Section 8, we consider the multispan Timoshenko beam model. AFMbased nanoscale processing with continuum surrounding media such as that found in biology and nanomachining applications [20-22] suggests observing frequency effects that arise with an academic two span beam model in which one segment includes rotatory inertia and shear deformation and the other one neglects both effects.

\section{Flexural Vibrations Using the Euler-Bernoulli Multispan Beam Model}

We consider a multispan microcantilever of length $L$ composed of $N$ segments in which the displacement $v_{j}(t, x)$ in the segment $x_{j-1} \leq x \leq x_{j}$ is governed by the Euler-Bernoulli model

$$
m_{j} \frac{\partial^{2} v_{j}(t, x)}{\partial t^{2}}+\mathrm{k}_{j} \frac{\partial^{4} v_{j}(t, x)}{\partial x^{4}}=f_{j}(t, x),
$$

where $m_{j}=\rho_{j} A_{j}$ is the mass per unit length, $A_{j}$ is the cross section area, $\rho_{j}$ is the mass density, $\mathrm{k}_{j}=E_{j} I_{j}$ is the flexural stiffness, and $f_{j}(t, x)$ is the transverse dynamic load at the $j-$ th station, $j=1: N$. Here $x_{0}=0$ and $X_{N}=L$.

2.1. Matrix Formulation. The stepped Euler-Bernoulli model (1) can be written as a second-order differential equation

$$
\mathrm{M} \ddot{\mathbf{v}}+\mathrm{Kv}=\mathrm{F},
$$

with diagonal matrix coefficients

$$
\begin{aligned}
& \mathrm{M}=\left(\begin{array}{ccc}
\mathrm{M}_{1} & & 0 \\
& \ddots & \\
0 & & \mathrm{M}_{N}
\end{array}\right), \\
& \mathrm{K}=\mathrm{EI} \frac{\partial^{4}}{\partial x^{4}}=\left(\begin{array}{ccc}
\mathrm{EI}_{1} & & 0 \\
& \ddots & \\
0 & & \mathrm{EI}_{N}
\end{array}\right) \frac{\partial^{4}}{\partial x^{4}}
\end{aligned}
$$

where

$$
\begin{aligned}
& \mathrm{M}_{j}=\rho_{j} A_{j}, \\
& \mathrm{EI}_{j}=E_{j} I_{j} .
\end{aligned}
$$


Here the displacement and the dynamic load $\mathrm{v}(t, x)$ and $\mathrm{F}(\mathrm{t}, \mathrm{x})$, at the multispan beam, are given in vector form as

$$
\begin{gathered}
\mathrm{v}(t, x)=\left(\begin{array}{c}
v_{1}(t, x) \\
v_{2}(t, x) \\
\vdots \\
v_{N}(t, x)
\end{array}\right), \\
\mathrm{F}=\left(\begin{array}{c}
f_{1}(t, x) \\
f_{2}(t, x) \\
\vdots \\
f_{N}(t, x)
\end{array}\right) .
\end{gathered}
$$

2.2. Boundary and Internal Conditions for a Multispan Cantilever Beam. For a cantilever beam, the boundary conditions can be written in a compact matrix form

$$
\begin{gathered}
\mathbf{B}_{0} \mathrm{v}_{0}=\mathbf{0}, \\
\mathbf{B}_{L} \mathrm{v}_{L}=\mathbf{0},
\end{gathered}
$$

where

$$
\begin{aligned}
& \mathbf{B}_{0}=\left(\begin{array}{llll}
1 & 0 & 0 & 0 \\
0 & 1 & 0 & 0
\end{array}\right), \\
& \mathbf{B}_{L}=\left(\begin{array}{llll}
0 & 0 & 1 & 0 \\
0 & 0 & 0 & 1
\end{array}\right), \\
& \mathrm{v}_{0}=\left(\begin{array}{c}
v_{1}(t, 0) \\
v_{1}^{\prime}(t, 0) \\
v_{1}^{\prime \prime}(t, 0) \\
v_{1}^{\prime \prime \prime}(t, 0)
\end{array}\right), \\
& \mathrm{v}_{L}=\left(\begin{array}{c}
v_{N}(t, L) \\
v_{N}^{\prime}(t, L) \\
v_{N}^{\prime \prime}(t, L) \\
v_{N}^{\prime \prime \prime}(t, L)
\end{array}\right) .
\end{aligned}
$$

At the points $x_{i}, i=1,2, \ldots, N-1$, located between two consecutive segments, the compatibility conditions for the displacement, slope, bending moment, and shear lead to the compatibility matrix relationship

$$
\mathscr{C}_{1, i} \mathrm{v}_{i}\left(t, x_{i}\right)=\mathscr{C}_{2, i} \mathrm{v}_{i+1}\left(t, x_{i}\right)
$$

with

$$
\begin{aligned}
\mathscr{C}_{1, i}= & \left(\begin{array}{llll}
1 & 0 & 0 & 0 \\
0 & 1 & 0 & 0 \\
0 & 0 & 1 & 0 \\
0 & 0 & 0 & 1
\end{array}\right), \\
\mathscr{C}_{2, i}= & \left(\begin{array}{llll}
1 & 0 & 0 & 0 \\
0 & 1 & 0 & 0 \\
0 & 0 & \alpha & 0 \\
0 & 0 & 0 & \alpha
\end{array}\right), \\
\mathrm{v}_{i} & =\left(\begin{array}{c}
v_{i}\left(t, x_{i}\right) \\
v_{i}^{\prime}\left(t, x_{i}\right) \\
v_{i}^{\prime \prime}\left(t, x_{i}\right) \\
v_{i}^{\prime \prime \prime}\left(t, x_{i}\right)
\end{array}\right),
\end{aligned}
$$

being $\alpha_{i}=E I_{i+1} / E I_{i}$ for $i=1, \ldots, N-1$.

\section{Forced Vibrations}

The dynamic response of a forced multispan Euler-Bernoulli model $\mathrm{M} \ddot{\mathbf{v}}+\mathbf{K v}=\mathrm{F}$ given in (2), subject to homogeneous boundary conditions, could be described in terms of the $N \times$ $N$ diagonal matrix impulse response or initial-value Green function $\mathbf{h}(t, x, \xi)$ satisfying the initial-value problem

$$
\begin{aligned}
\mathbf{M} \mathbf{h}(t, x, \xi)+\mathrm{Kh}(t, x, \xi) & =\mathbf{0} \\
0 & <x<L, 0<\xi<L, t>0, \\
\mathbf{h}(0, x, \xi) & =\mathbf{0} \\
\mathrm{Mh}_{t}(0, x, \xi) & =\delta(x-\xi) \mathbf{I}, \\
\mathbf{B}_{0} \mathrm{~h}_{0} & =\mathbf{0} \\
\mathbf{B}_{L} \mathrm{~h}_{L} & =\mathbf{0} \\
\mathscr{C}_{1, i} \mathrm{~h}_{i}\left(t, x_{i}^{-}, \xi\right) & =\mathscr{C}_{2, i} \mathrm{~h}_{i}\left(t, x_{i}^{+}, \xi\right)
\end{aligned}
$$

where I is the $N \times N$ identity matrix and $\mathscr{C}_{1, i}, \mathscr{C}_{2, i}, \mathrm{~h}_{\mathrm{i}}$ are as given in (10), by using the i-th diagonal component of $\mathbf{h}$.

To work on the frequency domain, we need to introduce transfer function $\mathbf{H}(s, x, \xi)$ as being the Laplace transform of $\mathbf{h}(t, x, \xi)$ with respect to time (For inputs $e^{\lambda t} F(x)$ we seek outputs $e^{\lambda t} V(x)$ where $V(x)=\mathrm{H} F(x)$ with $\mathrm{H}$ defined in (17)). Thus

$$
\begin{aligned}
\left(s^{2} \mathrm{M}+\mathrm{K}\right) \mathbf{H}(s, x, \xi) & =\delta(x-\xi) \mathbf{I}, \quad 0<x, \xi<L, \\
\mathbf{B}_{0} \mathrm{H}_{0} & =\mathbf{0}, \\
\mathbf{B}_{L} \mathrm{H}_{L} & =\mathbf{0}, \\
\mathscr{C}_{1, i} \mathrm{H}_{i}\left(t, x_{i}^{-}, \xi\right) & =\mathscr{C}_{2, i} \mathrm{H}_{i}\left(t, x_{i}^{+}, \xi\right) .
\end{aligned}
$$

It turns out that $\mathbf{h}(t-\tau, x, \xi)$ acts as an integrating factor in Lagrange's adjoint method for the forced equation (2). 
Multiplying (2) by $\mathbf{h}(t-\tau, x, \xi)$ and integrating by parts, we obtain the dynamic response

$$
\begin{aligned}
& \mathrm{v}(t, x)=\int_{0}^{t} \int_{0}^{L}\left(\mathbf{h}_{t}(\tau, x, \xi) \mathrm{Mv}_{o}(\xi)\right. \\
& \left.\quad+\mathbf{h}(\tau, x, \xi) \mathrm{Mv}_{1}(\xi)\right) d \xi d \tau+\int_{0}^{t} \int_{0}^{L} \mathbf{h}(t \\
& \quad-\tau, x, \xi) \mathrm{F}(\tau, \xi) d \xi d \tau,
\end{aligned}
$$

where $\mathrm{v}_{o}(x)=\mathrm{v}(0, x)$ and $\mathrm{v}_{1}(x)=\mathrm{v}_{t}(0, x)$ are given initial conditions.

The procedure mentioned above is also related to the Riemann function method for integrating partial differential equations. Dynamic responses have been considered in the field of control of distributed systems and in elastodynamics in connection with vibrations and cracking problems [2326].

In practice, when computing the convolution integral, which corresponds to the forced response $\mathrm{v}(t, x)$, with null initial values $\mathrm{v}(0, x)=0, \mathrm{v}_{t}(0, x)=0$, we actually have $\mathrm{v}(t, x)=\mathrm{v}_{h}(t, x)+\mathrm{v}_{p}(t, x)$, where $\mathrm{v}_{h}(t, x)$ is a free vibration introduced by the system, whose initial values are a priori unknown, and $\mathrm{v}_{p}(t, x)$ is a particular response. When $\mathrm{v}_{p}(t, x)$ can be determined by other means, then those initial values can be supplied as $\mathrm{v}_{h}(0, x)=-\mathrm{v}_{p}(0, x)$ and $\left(\mathrm{v}_{h}\right)_{t}(0, x)=$ $-\left(\mathrm{v}_{p}\right)_{t}(0, x)$ [27]. Thus, the induced system free response, due to a permanent response $\mathrm{v}_{p}(t, x)$, is given by

$$
\begin{aligned}
\mathrm{v}_{h}(t, x)= & -\int_{0}^{L} \mathbf{h}(t, x, \xi) \mathbf{M} \dot{\mathrm{v}}_{p}(0, \xi) d \xi \\
& -\int_{0}^{L} \frac{\partial \mathbf{h}(t, x, \xi)}{\partial t} \operatorname{Mv}_{p}(0, \xi) d \xi .
\end{aligned}
$$

3.1. Frequency Response. Harmonic and piecewise linear forcing are of interest in frequency analysis. When seeking a response of the same type, the transfer function is introduced. Given the harmonic input

$$
f(t, x)=e^{i \omega t} v(x)
$$

we have the harmonic output response

$$
\mathrm{v}_{p}(t, x)=e^{i \omega t} \mathrm{H}(i \omega) v(x)
$$

where

$$
\mathrm{H}(i \omega) v(x)=\int_{0}^{L} \mathbf{H}(i \omega, x, \xi) v(\xi) d \xi
$$

In particular, for a concentrated force at a point $x=a$, of spatial amplitude $\mathrm{v}(x)=v(x) \delta(x-a)$, we have the permanent response

$$
\mathrm{v}_{p}(t, x)=e^{i \omega t} \mathbf{H}(i \omega, x, a) v(a)
$$

With the initial values $v_{p}(0, \xi)=\mathbf{H}(i \omega, \xi, a) v(a), \dot{\mathrm{v}}_{p}(0, \xi)=$ $i \omega \mathrm{v}_{p}(0, \xi)$, the induced free response is given by

$$
\mathrm{v}_{h}(t, x)=-\int_{0}^{L} \mathrm{r}(t, x, \xi, \omega) \mathrm{MH}(i \omega, a, \xi) v(a) d \xi
$$

where

$$
\mathbf{r}=\mathbf{h}_{t}(t, x, \xi)+i \omega \mathbf{h}(t, x, \xi)
$$

For a pulse amplitude

$$
\mathrm{v}(x)=\mathrm{v}_{o}(u(x-a)-u(x-b)),
$$

where $u(x)$ is the unit step function, the permanent response turns out

$$
\mathrm{v}_{p}(t, x)=e^{i \omega t} \int_{a}^{b} \mathbf{H}(i \omega, x, \xi) \mathrm{v}_{o} d \xi
$$

As before, by substituting the initial values in (14), the induced free response will now be

$$
\mathrm{v}_{h}(t, x)=-\int_{0}^{L} \mathrm{r}(t, x, \xi, \omega) \mathrm{MH}(i \omega, x, \xi) \mathrm{v}(\xi) d \xi
$$

with $r$ given as in (20).

3.2. Closed Form of the Transfer Function for a Multispan Cantilever Beam. The Green function of a multispan EulerBernoulli can be determined by using an appropriate solution basis in each segment. From $(12), \mathbf{H}(s, x, \xi)$ is a diagonal matrix, with entries $H_{j}$ that satisfy the differential equation

$$
X^{(i v)}(x)-\varepsilon_{j}^{4} X(x)=\delta(x-\xi)
$$

where

$$
\varepsilon_{j}^{4}=-\frac{\rho_{j} A_{j} s^{2}}{E_{j} I_{j}}, \quad j=1,2, \cdots, N .
$$

The functions $\left\{h\left(x-x_{j-1}\right), h^{\prime}\left(x-x_{j-1}\right), h^{\prime \prime}\left(x-x_{j-1}\right), h^{\prime \prime \prime}(x-\right.$ $\left.x_{j-1}\right)$ \} constitute a solution basis of the equation $X^{(i v)}(x)-$ $\varepsilon_{j}^{4} X(x)=0$. Here

$$
h(x)=\frac{\sinh \left(\varepsilon_{j} x\right)-\sin \left(\varepsilon_{j} x\right)}{2 \varepsilon_{j}^{3}}
$$


is the solution of the initial-value problem

$$
\begin{aligned}
h^{(i v)}(x)-\varepsilon_{j}^{4} h(x) & =0, \\
h(0) & =0, \\
h^{\prime}(0) & =0, \\
h^{\prime \prime}(0) & =0, \\
h^{\prime \prime \prime}(0) & =1 .
\end{aligned}
$$

By denoting

$$
\begin{aligned}
& h_{0}(x)=h^{\prime \prime \prime}(x)=\frac{1}{2}\left(\cosh \varepsilon_{j} x+\cos \varepsilon_{j} x\right), \\
& h_{1}(x)=h^{\prime \prime}(x)=\frac{1}{2 \varepsilon}\left(\sinh \varepsilon_{j} x+\sin \varepsilon_{j} x\right), \\
& h_{2}(x)=h^{\prime}(x)=\frac{1}{2 \varepsilon_{j}^{2}}\left(\cosh \varepsilon_{j} x-\cos \varepsilon_{j} x\right), \\
& h_{3}(x)=h(x)=\frac{1}{2 \varepsilon_{j}^{3}}\left(\sinh \varepsilon_{j} x-\sin \varepsilon_{j} x\right),
\end{aligned}
$$

in each segment we have

$$
\mathbf{H}(s, x, \xi)=\left(\begin{array}{cccc}
H_{0} & 0 & \cdots & 0 \\
0 & H_{1} & \cdots & 0 \\
\vdots & \vdots & \vdots & \vdots \\
0 & 0 & \cdots & H_{N-1}
\end{array}\right) \text {, }
$$

for $j=0, . ., N-1$

$$
\begin{aligned}
H_{j}(s, x, \xi)= & h_{0}\left(x-x_{j}\right) H\left(s, x_{j}, \xi\right) \\
& +h_{1}\left(x-x_{j}\right) H_{x}\left(s, x_{j}, \xi\right) \\
& +h_{2}\left(x-x_{j}\right) H_{x x}\left(s, x_{j}, \xi\right) \\
& +h_{3}\left(x-x_{j}\right) H_{x x x}\left(s, x_{j}, \xi\right) \\
& +h_{3}\left(x-x_{j}\right) u(x-\xi),
\end{aligned}
$$

$$
x_{j} \leq x \leq x_{j+1}
$$

In order to determine $\mathbf{H}(s, x, \xi)$, we need to find the initialvalue vectors

$$
\mathrm{H}=\left(\begin{array}{c}
H\left(s, x_{j}, \xi\right) \\
H_{x}\left(s, x_{j}, \xi\right) \\
H_{x x}\left(s, x_{j}, \xi\right) \\
H_{x x x}\left(s, x_{j}, \xi\right)
\end{array}\right)
$$

By using the compatibility conditions

$$
\mathscr{C}_{1, i} \mathrm{H}\left(s, x_{j}^{-}, \xi\right)=\mathscr{C}_{2, i} \mathrm{H}\left(s, x_{j}^{+}, \xi\right),
$$

$$
j=1,2, \cdots, N-1,
$$

given similarly to (9), and by deriving (30) with respect to $x$.

The above process will be carried out for the case of a two stepped cantilever beam with the intermediate discontinuity point $x_{1}=l_{1}$.

The boundary conditions and the initial values of $h(x)$ lead to a simplification of $H$ in the first and last segments. We have two null initial values at $x=0$, and we can use the boundary conditions at $x=L$ as initial values, instead of those at $x_{N-1}$. This is possible by changing the solution basis at the last segment in (30) to the one generated by $h(L-x)$.

Thus, for $0 \leq \xi \leq L$

$$
\begin{aligned}
H(s, x, \xi)= & h_{2}(x) H_{x x}(s, 0, \xi)+h_{3}(x) H_{x x x}(s, 0, \xi) \\
& +h_{3}(x-\xi) u(x-\xi), \quad 0 \leq x \leq x_{1}, \\
H(s, x, \xi)= & h_{0}(L-x) H(s, L, \xi) \\
& +h_{1}(L-x) H_{x}(s, L, \xi) \\
& +h_{3}(\xi-x) u(\xi-x), \quad x_{1} \leq x \leq L .
\end{aligned}
$$

By applying the compatibility conditions

$$
\begin{aligned}
H\left(s, l_{1}^{-}, \xi\right) & =H\left(s, l_{1}^{+}, \xi\right), \\
H_{x}\left(s, l_{1}^{-}, \xi\right) & =H_{x}\left(s, l_{1}^{+}, \xi\right), \\
E_{1} I_{1} H_{x x}\left(s, l_{1}^{-}, \xi\right) & =E_{2} I_{2} H_{x x}\left(s, l_{1}^{+}, \xi\right), \\
E_{1} I_{1} H_{x x x}\left(s, l_{1}^{-}, \xi\right) & =E_{2} I_{2} H_{x x x}\left(s, l_{1}^{+}, \xi\right),
\end{aligned}
$$

we get the algebraic system

$$
\left(\begin{array}{cccc}
h_{2}\left(l_{1}\right) & h_{3}\left(l_{1}\right) & -\left.\left(h_{0}(L-x)\right)\right|_{x=l_{1}} & -\left.\left(h_{1}(L-x)\right)\right|_{x=l_{1}} \\
h_{2}^{\prime}\left(l_{1}\right) & h_{3}^{\prime}\left(l_{1}\right) & -\left.\left(h_{0}^{\prime}(L-x)\right)\right|_{x=l_{1}} & -\left.\left(h_{1}^{\prime}(L-x)\right)\right|_{x=l_{1}} \\
h_{2}^{\prime \prime}\left(l_{1}\right) & h_{3}^{\prime \prime}\left(l_{1}\right) & -\left.\left(\frac{E_{2} I_{2}}{E_{1} I_{1}} h_{0}^{\prime \prime}(L-x)\right)\right|_{x=l_{1}} & -\left.\left(\frac{E_{2} I_{2}}{E_{1} I_{1}} h_{1}^{\prime \prime}(L-x)\right)\right|_{x=l_{1}} \\
h_{2}^{\prime \prime \prime}\left(l_{1}\right) & h_{3}^{\prime \prime \prime}\left(l_{1}\right) & -\left.\left(\frac{E_{2} I_{2}}{E_{1} I_{1}} h_{0}^{\prime \prime \prime}(L-x)\right)\right|_{x=l_{1}} & -\left(\frac{E_{2} I_{2}}{E_{1} I_{1}} h_{1}^{\prime \prime \prime}(L-x)\right)
\end{array}\right)\left(\begin{array}{c}
H^{\prime \prime}(s, 0, \xi) \\
H^{\prime \prime \prime}(s, 0, \xi) \\
H(s, L, \xi) \\
H^{\prime}(s, L, \xi)
\end{array}\right)
$$




$$
=\left(\begin{array}{c}
h\left(l_{1}-\xi\right) u\left(l_{1}-\xi\right)-h\left(l_{1}-\xi\right) u\left(l_{1}-\xi\right) \\
h^{\prime}\left(l_{1}-\xi\right) u\left(l_{1}-\xi\right)-h^{\prime}\left(l_{1}-\xi\right) u\left(l_{1}-\xi\right) \\
\left.\frac{E_{2} I_{2}}{E_{1} I_{1}}\left(h^{\prime \prime}(\xi-x)\right)\right|_{x=l_{1}} u\left(\xi-l_{1}\right)-h^{\prime \prime}\left(l_{1}-\xi\right) u\left(l_{1}-\xi\right) \\
\left.\frac{E_{2} I_{2}}{E_{1} I_{1}}\left(h^{\prime \prime \prime}(\xi-x)\right)\right|_{x=l_{1}} u\left(\xi-l_{1}\right)-h^{\prime \prime \prime}\left(l_{1}-\xi\right) u\left(l_{1}-\xi\right)
\end{array}\right),
$$

for determining $H_{j}(s, x, \xi)$ from (30).

Remark. The case of axially loaded stepped beams has been investigated in [28] with nonclassical conditions. There, the Green function was obtained by working with a solution basis that considers hyperbolic and trigonometric functions in the general solution. These functions depend upon simple roots of a complete quadratic polynomial due to the inclusion of a second derivative spatial term in (24). When the axial load is removed, the solution basis reduces to the one considered for particular cases of vibrating beams. We observe that through a limit process the use of $h(x)$, which involves a division by root parameters, can also handle the case of repeated roots, which is of interest in the static case or critical frequencies.

\section{A Cantilever Beam with a Piezoelectric Layer in AFM}

Active microcantilever beams due to their structural flexibility and sensitivity to atomic and molecular forces have received increased attention in a variety of nanoscale sensing and measuring applications, including atomic force microscopy, thermal scanning microscopy, and biomass sensing.

In [9], a microcantilever model was proposed for studying atomic force microscope. It was formulated as a three stepped beam with a piezoelectric layer patch in the first segment and the other two segments were simple beams with different cross-sectional areas according to Figure 1(a).

The governing equations can be written as a system of three second-order partial differential equations with constant coefficients

$$
\begin{array}{ll}
m_{1} \frac{\partial^{2} u_{1}(t, x)}{\partial t^{2}}+(E I)_{1} \frac{\partial^{4} u_{1}(t, x)}{\partial x^{4}}= & \frac{\partial^{2} M_{p}(t, x)}{\partial x^{2}}, \\
& 0 \leq x \leq l_{1}, \\
m_{2} \frac{\partial^{2} u_{2}(t, x)}{\partial t^{2}}+(E I)_{2} \frac{\partial^{4} u_{2}(t, x)}{\partial x^{4}}=0, & l_{1}<x \leq l_{2}, \\
m_{3} \frac{\partial^{2} u_{3}(t, x)}{\partial t^{2}}+(E I)_{3} \frac{\partial^{4} u_{3}(t, x)}{\partial x^{4}}=0 & l_{2}<x \leq L,
\end{array}
$$

subject to cantilever boundary conditions (7) and compatibility conditions (9) at the discontinuity points $x_{1}, x_{2}$. The parameters $m_{j}$ and $(E I)_{j}$ for each segment of the beam are given by

$$
\begin{aligned}
& m_{1}=(\rho A)_{1}=\rho_{p} w_{p} t_{p}+\rho_{b} w_{b 1} t_{b} \\
& m_{2}=(\rho A)_{2}=\rho_{b} w_{b 1} t_{b} \\
& m_{3}=(\rho A)_{3}=\rho_{b} w_{b 2} t_{b} \\
& (E I)_{1}=E_{p} I_{p p}+E_{b} I_{p b}+E_{b} I_{b 1} \\
& (E I)_{2}=E_{b} I_{b 1} \\
& (E I)_{3}=E_{b} I_{b 2} .
\end{aligned}
$$

The parameters $\rho_{p}, \rho_{b}$ are the mass density of piezoelectric material and mass density of beam material; $w_{p}, w_{b 1}$, and $w_{b 2}$ are the piezoelectric layer width, the width of the second, and third beam segments, respectively; $t_{p}$ and $t_{b}$ are the piezoelectric layer thickness and beam thickness. $E_{p}$ and $E_{b}$ are the Young modulus of piezoelectric layer material and Young modulus of second and third segment material. $I_{b 1}$, $I_{b 2}, I_{p p}, I_{p b}$ are the beam moment of inertia, layer moment of inertia, and layer-beam moment of inertia and are calculated as $I_{b 1}=w_{b 1} t_{b}^{3} / 12, I_{b 2}=w_{b 2} t_{b}^{3} / 12, I_{p p}=z_{n}^{2} t_{p} w_{p}+\left((1 / 3) t_{p}^{3}+\right.$ $\left.(1 / 2) t_{b} t_{p}^{2}+(1 / 4) t_{b}^{2} t_{p}\right) w_{p}-z_{n}\left(t_{p}^{2}+t_{b} t_{p}\right) w_{p} ; I_{p b}=z_{n}^{2} t_{b} w_{b 1}$. And $z_{n}$ is the neutral axis of the beam on the composite portion expressed by

$$
z_{n}=\frac{1}{2} \frac{E_{p} t_{p} w_{p}\left(t_{p}+t_{b}\right)}{E_{p} t_{p} w_{p}+E_{b} t_{b} w_{b 1}} .
$$

The values of these parameters [9] are presented in Table 1. The distributed cross-sectional moment $M_{p}(x, t)=$ $M_{p o}(t)\left(1-u\left(x-l_{1}\right)\right)$ in the first segment induces a forcing term. Its time amplitude is given by

$$
M_{p o}(t)=\frac{1}{2} w_{p} E_{p} d_{31}\left(t_{b}+t_{p}\right) v(t)
$$

where $d_{31}$ and $v(t)$ are the coefficients of the converse piezoelectric effect and the applied voltage, respectively. 


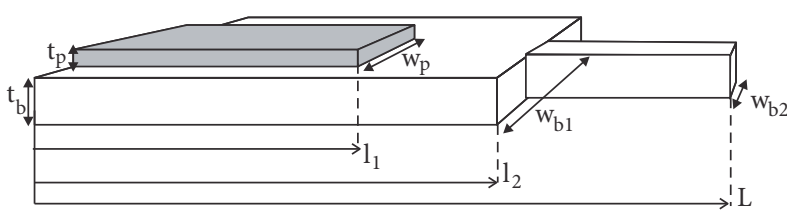

(a)

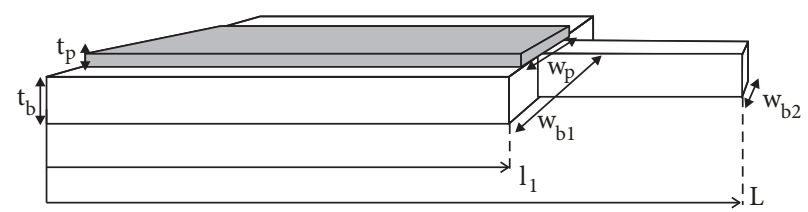

(b)

FIGURE 1: Schematic representation of the (a) triple span and (b) double-span piezoelectric beams.

4.1. Forced Responses. For homogeneous boundary conditions and null initial data, we have from (13) the forced response

$$
\begin{aligned}
& \mathrm{v}(\mathrm{t}, \mathrm{x})=\int_{0}^{\mathrm{t}} \int_{0}^{\mathrm{L}} \mathbf{h}(\mathrm{t}-\tau, \mathrm{x}, \xi) \mathrm{F}(\tau, \xi) \mathrm{d} \xi \mathrm{d} \tau \\
& \mathrm{F}(\mathrm{t}, \mathrm{x})=\left(\begin{array}{c}
\frac{\partial^{2} M_{p}(t, x)}{\partial x^{2}} \\
0 \\
0
\end{array}\right),
\end{aligned}
$$

where $\partial^{2} M_{p}(\tau, \xi) / \partial \xi^{2}=-M_{p o}(\tau) \delta^{\prime}\left(\xi-l_{1}\right)$. Thus

$$
\begin{aligned}
\mathrm{v}(\mathrm{t}, \mathrm{x}) & =\int_{0}^{t} \int_{0}^{L} \mathbf{h}(t-\tau, x, \xi) \frac{\partial^{2} M_{p}(\tau, \xi)}{\partial \xi^{2}} d \xi d \tau \\
& =\int_{0}^{t} M_{p o}(\tau) \frac{\partial \mathbf{h}}{\partial \xi}\left(t-\tau, x, l_{1}\right) d \tau .
\end{aligned}
$$

$$
X^{(n)}(x, \xi)=\left(\begin{array}{cccc}
X_{1}^{(n)}(x) X_{1}^{(n)}(\xi) & 0 & \cdots & 0 \\
0 & X_{2}^{(n)}(x) X_{2}^{(n)}(\xi) & \cdots & 0 \\
\vdots & \vdots & \vdots & \vdots \\
0 & 0 & \cdots & X_{N-1}^{(n)}(x) X_{N-1}^{(n)}(\xi)
\end{array}\right) .
$$

is the spatial amplitude of exponential solutions $\mathrm{v}(t, x)=$ $e^{\lambda t} X(x)$ of the unforced Euler-Bernoulli model M $\ddot{\mathbf{v}}+\mathrm{K} \mathbf{v}=0$ that satisfy the boundary conditions (6), and the compatibility conditions (9) at the discontinuity points $x_{1}$ and $x_{2}$.

The modal approximation

$$
\frac{\partial \mathbf{h}}{\partial \xi}(t-\tau, x, \xi)=\sum_{n=1}^{m} \frac{\partial X^{(n)}}{\partial \xi}(x, \xi) \frac{\sin \omega_{n}(t-\tau)}{\omega_{n}}
$$

By assuming a discrete complete spectrum of natural frequencies $\omega_{j}$ and the normal mode property, the Galerkin method can be used for determining approximate forced vibrations. We have the modal series

$$
\mathbf{h}(t, x, \xi)=\sum_{n=1}^{\infty} X^{(n)}(x, \xi) \frac{\sin \omega_{n} t}{\omega_{n}}, \quad 0 \leq x, \xi \leq L
$$

and the spatial Green function

$$
\mathbf{H}(t, x, \xi)=\sum_{n=1}^{\infty} X^{(n)}(x, \xi) \frac{1}{s^{2}+\omega_{n}^{2}}
$$

where

has been used in simulations with and without the inclusion of a piezoelectric layer. Expansions were truncated with a small number $m$ of terms, usually between 5 and 10 [30].

In Table 2 are showed the first six natural frequencies obtained in this work for a triple span beam with piezoelectric patch and without it. The first three frequencies are compared with those obtained experimentally in [9]. There is an agreement with the methodology of this work and it can be observed that the elimination of piezoelectric layers diminishes the frequency values. In Figure 2 are shown the corresponding vibration modes; in the first segment the modes of piezoelectric beam have smaller amplitude.

The effects of the inclusion of a piezoelectric patch are appreciated by applying a harmonic rectangular pulse load in the first segment and in the third segment for a three stepped beam with and without piezoelectric layer. It is observed 
TABLE 1: Parameters of piezoelectric multispan beam.

\begin{tabular}{lcc}
\hline Parameter & Numeric Value & Unity \\
\hline Length $L$ & $486 \times 10^{-6}$ & $\mathrm{~m}$ \\
Length $l_{1}$ & $325 \times 10^{-6}$ & $\mathrm{~m}$ \\
Length $l_{2}$ & $360 \times 10^{-6}$ & $\mathrm{~m}$ \\
Width $w_{b 1}$ & $250 \times 10^{-6}$ & $\mathrm{~m}$ \\
Width $w_{b 2}$ & $55 \times 10^{-6}$ & $\mathrm{~m}$ \\
Width $w_{p}$ & $130 \times 10^{-6}$ & $\mathrm{~m}$ \\
Thickness $t_{p}$ & $4 \times 10^{-6}$ & $\mathrm{~m}$ \\
Thickness $t_{b}$ & $4 \times 10^{-6}$ & $\mathrm{~m}$ \\
Density $\rho_{b}$ & 2330 & $\mathrm{Kg} / \mathrm{m}^{3}$ \\
Density $\rho_{p}$ & 6390 & $\mathrm{Kg} / \mathrm{m}^{3}$ \\
Young's modulus $E_{b}$ & $\mathrm{GPa}$ \\
Young's modulus $E_{p}$ & 105 & $\mathrm{GPa}$ \\
\hline
\end{tabular}

TABLE 2: Comparative natural frequencies between cases of double and triple span beams with and without piezoelectric patch on the first segment.

\begin{tabular}{|c|c|c|c|c|c|c|c|}
\hline \multirow[t]{2}{*}{ Case } & \multirow[t]{2}{*}{ Piezoelectric path } & \multicolumn{6}{|c|}{ Natural frequencies $\omega_{i}(\mathrm{~Hz})$} \\
\hline & & $\omega_{1}$ & $\omega_{2}$ & $\omega_{3}$ & $\omega_{4}$ & $\omega_{5}$ & $\omega_{6}$ \\
\hline Triple span [9]* & with piezoelectric path & 52.3 & 203.0 & 382.5 & - & - & - \\
\hline Triple span (Figure 1(a)) & with piezoelectric path & 50.6 & 209.2 & 393.7 & 910.7 & 1412.5 & 2016.7 \\
\hline Double span (Figure 1(b)) & with piezoelectric path & 46.2 & 222.9 & 376.2 & 875.3 & 1537.5 & 1948.6 \\
\hline Double/Triple span & without piezoelectric path & 27.3 & 142.9 & 320.1 & 618.9 & 1073.0 & 1579.4 \\
\hline
\end{tabular}

that the spatial amplitude diminishes with the inclusion of a piezoelectric patch when the pulse is positioned in the piezoelectric layer segment; this effect does not appear when the pulse is positioned in the third segment (Figure 3).

In Figure 4 are compared the forced responses due to forcing terms only in the first segment. The first output tracks the forcing input $f_{1}(t, x)=M_{p 0}(t)\left(1-u\left(x-l_{1}\right)\right)$. For the original forcing term proposed in [9] $f_{1}(t, x)=$ $\partial^{2} M_{p}(t, x) / \partial x^{2}=-M_{p 0}(t) \delta^{\prime}\left(x-l_{1}\right)$, we can observe that the effect of doublet due to derivation of Heaviside function is to concentrate the output at the point $x=l_{1}$. Also, the amplitudes of forced response are smaller for three stepped beam with piezoelectric layer.

\section{Double-Span Microbeam with Material and Geometric Discontinuities}

We consider a double-span beam of length $L=486 \times 10^{-6} \mathrm{~m}$, formed for two segments with different rectangular transversal section area, according to Figure 1(b). For simulation purposes, we consider the same parameters utilized in [9] for the three stepped beam with a piezoelectric patch in the first segment, and now for the double-span beam, obtained by removing the second segment of the first three stepped beam, which means extending the piezoelectric layer from $l_{1}$ to $l_{2}$. In our methodology, this amounts to consider the limit case when $l_{1}$ tends to $l_{2}$, so that the flexural stiffness $(E I)_{1}$ and mass density $(\rho A)_{1}$ will be valid on the first segment $\left[0, l_{2}\right]$, since $l_{1}$ was extended to $l_{2}$, and on the new second segment $\left[l_{2}, L\right]$ we will have $(E I)_{3}$ and $(\rho A)_{3}$, described in (37) and (38).

The first six natural frequencies of this beam, with and without piezoelectric patch, were given in the third and fourth rows of Table 2 and were obtained by the modal method. We observed that the reduction of three segments for two segments, maintaining the others material and geometric properties, influences the magnitude of the natural frequencies. When the piezoelectric patch is removed, then the frequencies also decrease. The results were the same as those of the case of three stepped beam when removing the piezoelectric patch.

In Figure 5, by using the methodology described from (24) to (35), is presented a 3D graphic of the Green function $H(s, x, \xi)$ of the two stepped piezoelectric beam, for the sixth natural frequency. The amplitude of this Green function has peaks at the same natural frequencies obtained by the modal method (Table 2, third row).

\section{Surface Elasticity and Residual Surface Tension in the Timoshenko Beam Model}

The Timoshenko beam model has been modified with the inclusion of the residual tension and surface elastic modulus [14]. By using the energy method with the elastic strain 

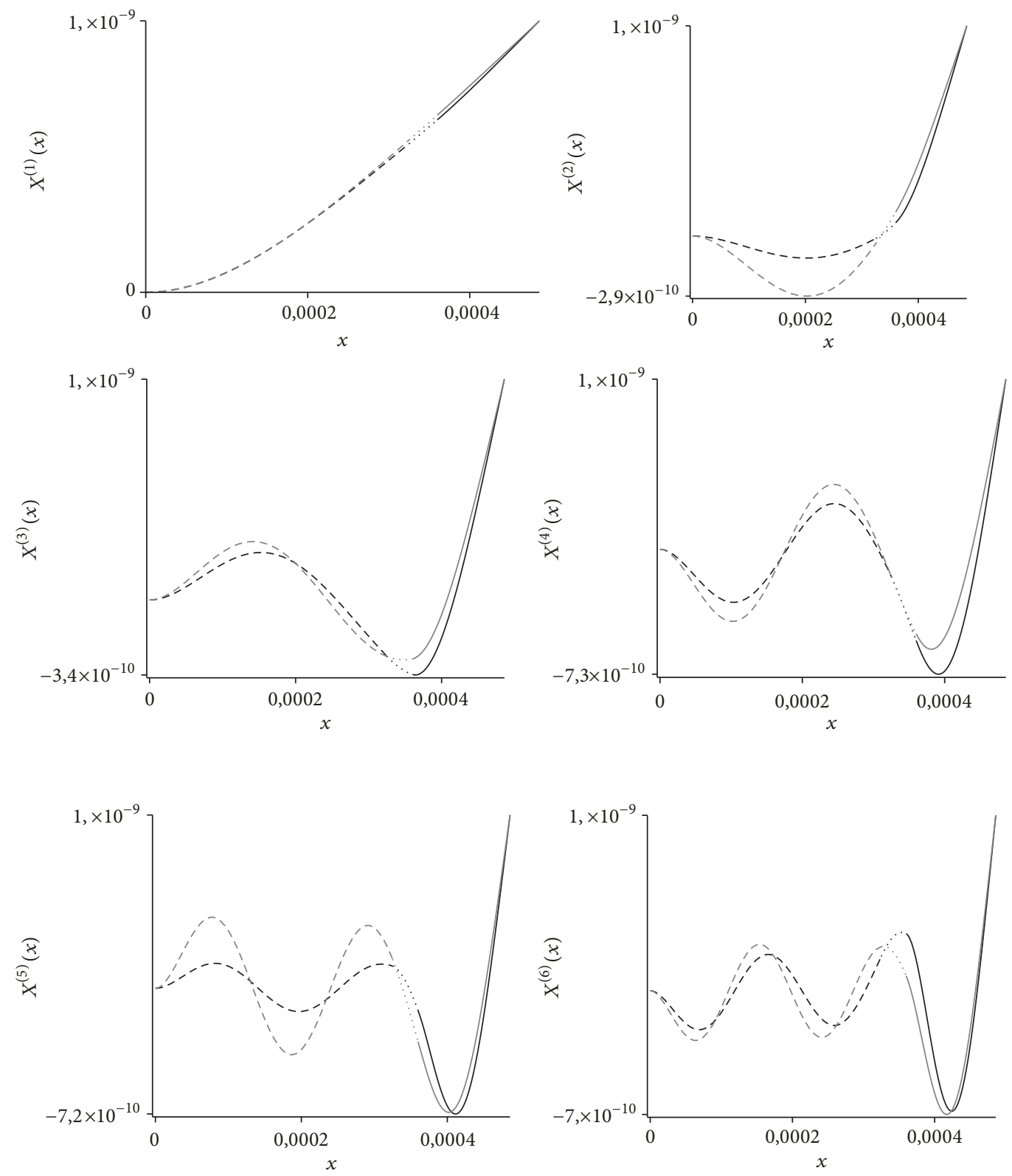

FiguRE 2: First six beam vibration modes: beam with piezoelectric layer (black line); beam (gray line) 1 st segment $\left(\right.$ dash) $\left[0, l_{1}\right], l_{1}=325 \times$ $10^{-6} \mathrm{~m}$; 2nd segment (dot) $\left[l_{1}, l_{2}\right], l_{2}=360 \times 10^{-6} \mathrm{~m}$; 3 rd segment (solid) $\left[l_{2}, L\right], L=486 \times 10^{-6} \mathrm{~m}$.

energy of surface induced in the potential energy and residual surface tensions, expressed by Laplace-Young equations, it was obtained the modified Timoskenko model

$$
\begin{aligned}
\rho A w_{t t}-\kappa G A w_{x x}+\overline{\kappa G A} \psi_{x} & =f(t, x), \\
\rho I \psi_{t t}-\overline{E I} \psi_{x x}-\kappa G A\left(w_{x}-\psi\right) & =q(t, x),
\end{aligned}
$$

for the flexural deflection $w(t, x)$ of the beam and the rotation angle $\psi(t, x)$ of the cross section of the beam. Here $f(t, x)$ denotes a transverse dynamic load and $q(t, x)$ a moment load.
The values

$$
\begin{aligned}
\overline{\kappa G A} & =\kappa G A-\left(\tau_{u}+\tau_{b}\right) b, \\
\overline{E I} & =\left(E I+2 b h^{2} E_{s}\right)
\end{aligned}
$$

are the effective curvature effect and flexural rigidity, respectively. Here $\tau_{u}$ and $\tau_{b}$ denote the upper and lower surfaces residual tensions and $E_{s}$ is a surface elastic modulus. Without these later parameters, the model (48) that includes surface effects becomes the classical Timoshenko model. As usual, Young's modulus, moment of inertia of the cross section area, shear modulus, mass density area, and shear deformation 

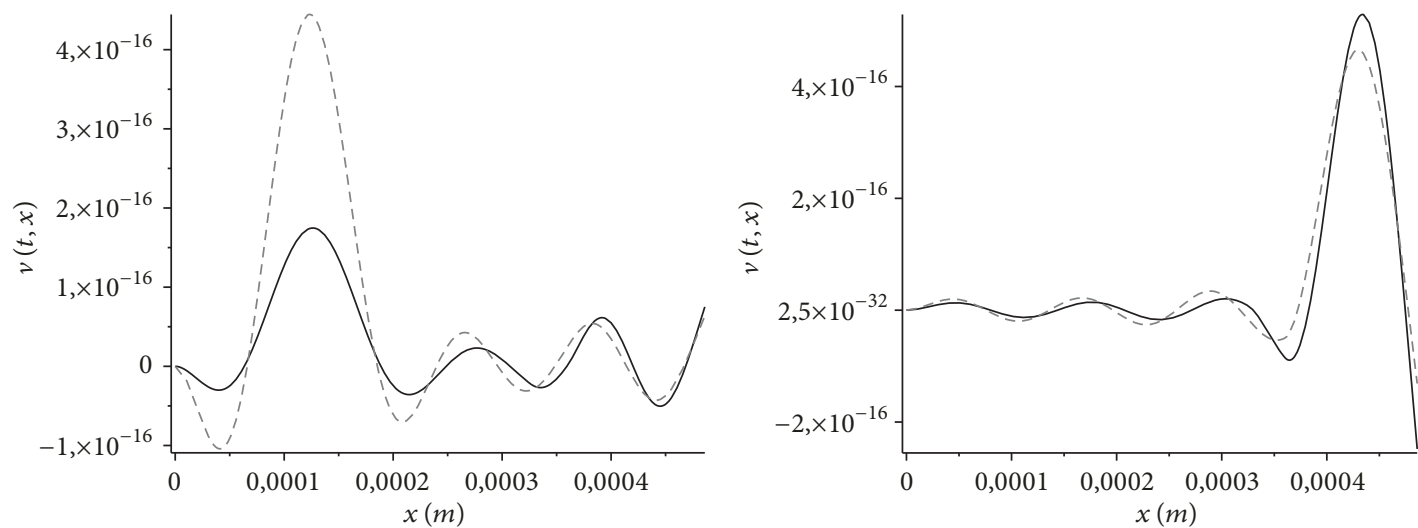

FIGURE 3: Left: forced response $\mathrm{v}(\mathrm{t}, \mathrm{x})$ with fixed $t$ for $\mathrm{f}_{1}(\mathrm{x})=\sin (\omega \mathrm{t})(\mathrm{u}(\mathrm{x}-\mathrm{a})-\mathrm{u}(\mathrm{x}-\mathrm{b})), a=100 \times 10^{-6} m$, and $b=150 \times 10^{-6} m$. Right: forced response $v(t, x)$ with fixed $t$ for $f_{3}(t, x)=\sin (\omega t)(u(x-c)-u(x-d)), c=430 \times 10^{-6} \mathrm{~m}$, and $d=440 \times 10^{-6} \mathrm{~m}$. Solid black line: beam with piezoelectric layer. Dashed gray line: beam without piezoelectric layer.
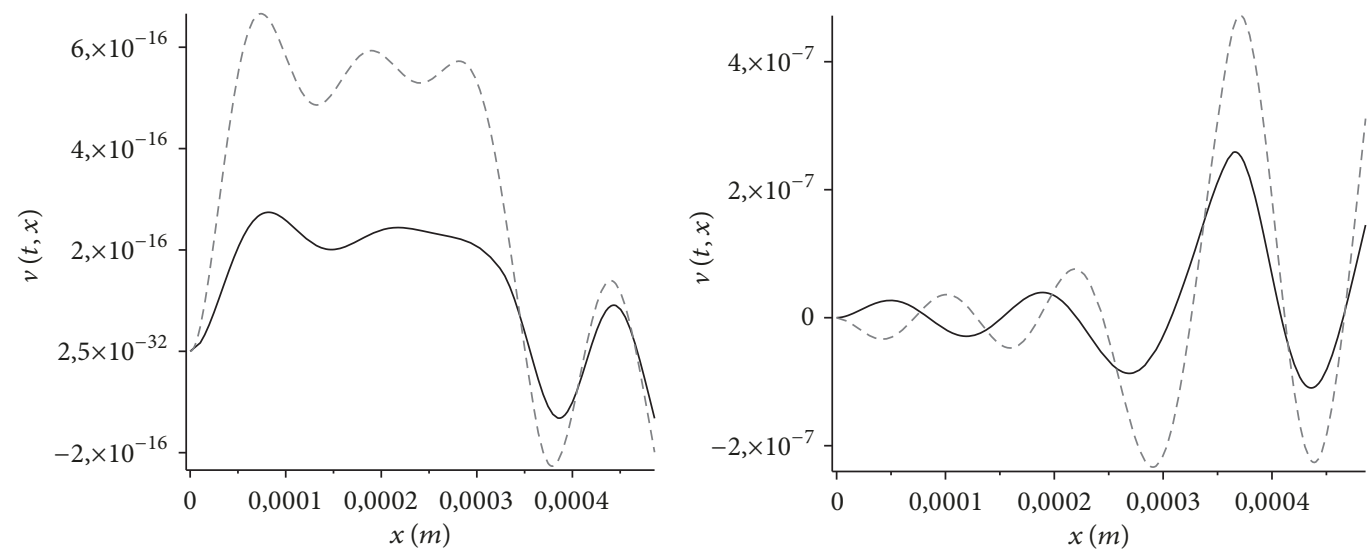

FIGURE 4: Left: forced response $v(t, x)$ for $f_{1}(t, x)=v(t)\left(1-u\left(x-l_{1}\right)\right)$. Right: forced response $v(t, x)$ for $f_{1}(t, x)=v(t)\left(\partial^{2} M_{p}(x) / \partial x^{2}\right)$. Beam without piezoelectric layer (dashed line) and beam with piezoelectric layer (solid line), $v(t)=\sin (\omega t)$.
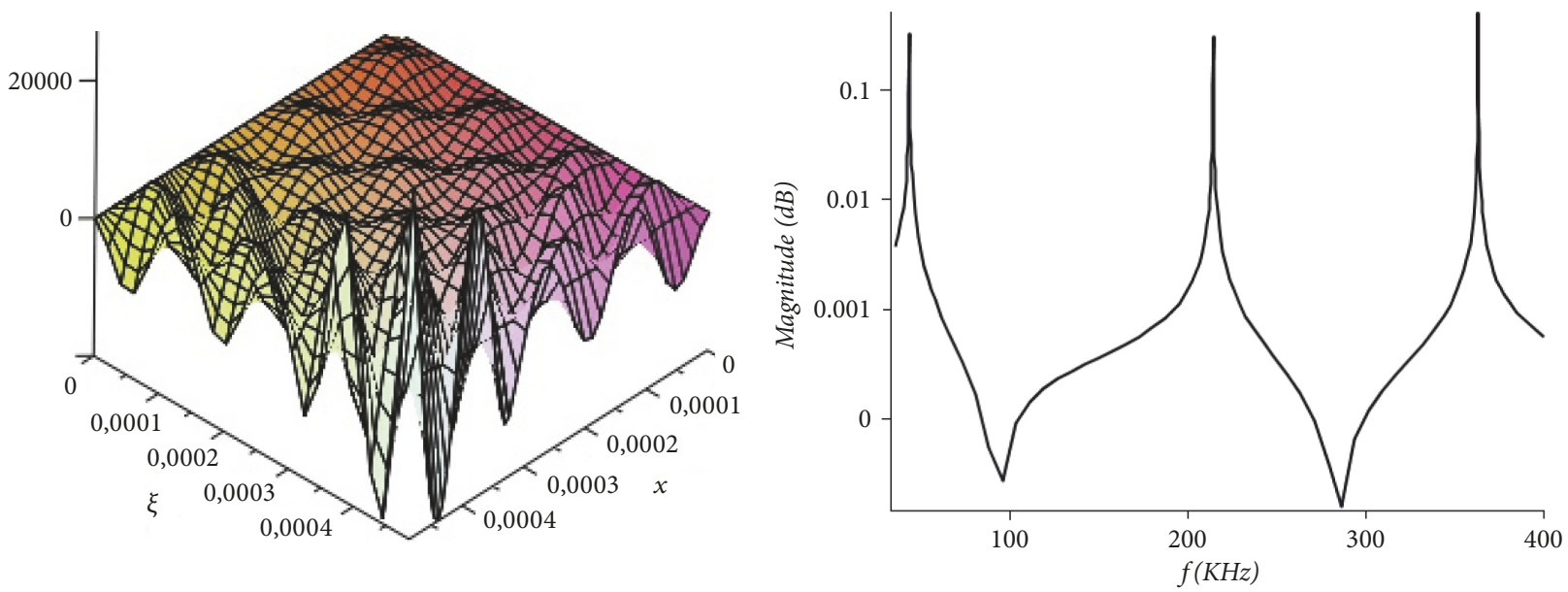

FIGURE 5: Left: frequency function $H\left(i \omega_{6}, x, \xi\right)$. Right: modal frequency response $H\left(i \omega, x_{o}, \xi_{o}\right)$ with the first three natural frequencies: $46.2 \mathrm{KHz}, 222.9 \mathrm{KHz}$, and $376.2 \mathrm{Khz}$ at a given point. 
factor of the beam are denoted as $E, I, G, \rho$, and $\kappa$, respectively. It is assumed that the beam has length $L$ and area $A=2 b h$, where $b, 2 h$ are the width and thickness of the beam and $I=2 b h^{3} / 3$. The boundary conditions are those of a cantilever beam or subject to balance of the moment and shear at the free end $x=L[31]$.

6.1. Matrix Formulation. The coupled Timoshenko model (48) can be written as a second-order differential equation with matrix coefficients

$$
M \ddot{v}+K v=F,
$$

where

$$
\begin{aligned}
& \mathbf{v}=\left(\begin{array}{l}
w(t, x) \\
\psi(t, x)
\end{array}\right), \\
& \mathrm{F}=\left(\begin{array}{l}
f(t, x) \\
q(t, x)
\end{array}\right), \\
& \mathrm{M}=\left(\begin{array}{ll}
\rho A & 0 \\
0 & \rho I
\end{array}\right), \\
& \mathrm{K}=\mathrm{K}_{2} \frac{\partial^{2}}{\partial x^{2}}+\mathrm{K}_{1} \frac{\partial}{\partial x}+\mathrm{K}_{0},
\end{aligned}
$$

with

$$
\begin{aligned}
& \mathrm{K}_{2}=\left(\begin{array}{cc}
-\kappa G A & 0 \\
0 & -\overline{E I}
\end{array}\right), \\
& \mathrm{K}_{1}=\left(\begin{array}{cc}
0 & \overline{\kappa G A} \\
-\kappa G A & 0
\end{array}\right), \\
& \mathrm{K}_{0}=\left(\begin{array}{cc}
0 & 0 \\
0 & \kappa G A
\end{array}\right) .
\end{aligned}
$$

For a microcantilever beam of length $L$, we have the boundary conditions in a matrix formulation

$$
\begin{gathered}
\left(\begin{array}{ll}
1 & 0 \\
0 & 1
\end{array}\right) \mathrm{v}(t, 0)+\left(\begin{array}{ll}
0 & 0 \\
0 & 0
\end{array}\right) \mathrm{v}_{x}(t, 0)=0, \\
\left(\begin{array}{cc}
0 & 0 \\
0 & -1
\end{array}\right) \mathrm{v}(t, L)+\left(\begin{array}{ll}
0 & 1 \\
1 & 0
\end{array}\right) \mathrm{v}_{x}(t, L)=0,
\end{gathered}
$$

or in a more compact form

$$
\begin{aligned}
& \mathrm{Av}(t, 0)+\mathrm{Bv}_{x}(t, 0)=0, \\
& \mathrm{Pv}(t, L)+\mathrm{Qv}_{x}(t, L)=0 .
\end{aligned}
$$

6.2. Eigenanalysis. The search of exponential solutions

$$
\begin{aligned}
\mathbf{v}(t, x) & =e^{\lambda t} \mathbf{v}(x), \\
\mathbf{v}(x) & =\left(\begin{array}{l}
w(x) \\
\psi(x)
\end{array}\right),
\end{aligned}
$$

of the unforced Timoshenko model

$$
M \frac{\partial^{2} v}{\partial t^{2}}+K v=0
$$

subject to general separated homogeneous boundary conditions (54), results in that $\mathbf{v}$ is the general solution of a secondorder matrix differential equation and is given by

$$
\mathbf{v}(x)=\mathbf{h}(x) \mathbf{c}_{1}+\mathbf{h}^{\prime}(x) \mathbf{c}_{2},
$$

for constant $2 \times 1$ vectors $\mathbf{c}_{1}$ and $\mathbf{c}_{2}$. Here $\mathbf{h}(x)$ is the $2 \times 2$ matrix solution of the initial-value problem

$$
\begin{aligned}
M \mathbf{h}^{\prime \prime}(x)+C \mathbf{h}^{\prime}(x)+K(\lambda) \mathbf{h}(x) & =\mathbf{0}, \\
\mathbf{h}(0) & =\mathbf{0}, \\
M \mathbf{h}^{\prime}(0) & =\mathbf{I},
\end{aligned}
$$

where $\mathbf{0}$ denotes the $2 \times 2$ null matrix and $\mathbf{I}$ the $2 \times 2$ identity matrix. The matrix coefficients are

$$
\begin{aligned}
M & =\left(\begin{array}{cc}
-\kappa G A & 0 \\
0 & -\overline{E I}
\end{array}\right), \\
C & =\left(\begin{array}{cc}
0 & \overline{\kappa G A} \\
-\kappa G A & 0
\end{array}\right), \\
K(\lambda) & =\left(\begin{array}{cc}
\rho A \lambda^{2} & 0 \\
0 & \lambda^{2} \rho I+\kappa G A
\end{array}\right) .
\end{aligned}
$$

By using the initial values of $\mathbf{h}(\mathrm{x})$ in (57) and the clamped boundary condition $\mathbf{v}(0)=\mathbf{0}, \mathrm{b}$, it turns out that $\mathbf{c}_{2}=\mathbf{0}$. Thus we have to determine $\lambda$ so that

$$
\mathbf{v}(x)=\mathbf{h}(x, \lambda) \mathbf{c}_{\mathbf{1}}
$$

satisfies the boundary condition at the free end $x=L$. By assuming homogeneous boundary conditions, we have the nonlinear eigenmatrix problem

$$
\mathbf{U}(\lambda) \mathbf{c}=\left(\mathrm{Ph}(L, \lambda)+\mathrm{Qh}^{\prime}(L, \lambda)\right) \mathbf{c}_{\mathbf{1}}=\mathbf{0} .
$$

From this, it turns out the characteristic equation

$$
\Delta(\lambda)=\operatorname{det}(\mathbf{U})=\mathbf{0} .
$$

We thus have the reduced system

$$
\begin{aligned}
\mathcal{U}_{D} \mathbf{c}_{1} & =\mathbf{0}, \\
\mathbf{c}_{1} & =\left(\begin{array}{c}
c_{11} \\
c_{12}
\end{array}\right)^{T},
\end{aligned}
$$

with

$$
\begin{aligned}
& \mathscr{U}_{D} \\
& =\left(\begin{array}{cc}
a d^{\prime \prime}(L) & -a d^{\prime \prime \prime}(L)+\lambda^{2} c d^{\prime}(L) \\
-b_{m} d^{\prime \prime \prime}(L)+\lambda^{2} e d^{\prime}(L) & a d^{\prime \prime}(L)-\lambda^{2} c d(L)-a_{m} d^{\prime \prime}(L)
\end{array}\right) .
\end{aligned}
$$


where

$$
d(x)=\frac{\delta \operatorname{senh}(\varepsilon x)-\varepsilon \operatorname{sen}(\delta x)}{a b_{m} \varepsilon \delta\left(\delta^{2}+\varepsilon^{2}\right)}
$$

is the solution of the initial-value problem

$$
\begin{aligned}
& a b_{m} d^{(i v)}(x)+\left(-a e \lambda^{2}-c \lambda^{2} b_{m}-a^{2}+a_{m} a\right) d^{\prime \prime}(x) \\
& \quad+\left(c \lambda^{2} a+c \lambda^{4} e\right) d(x)=0 \\
& d(0)=d^{\prime}(0)=d^{\prime \prime}(0)=0 \\
& a b_{m} d^{\prime \prime \prime}(0)=1
\end{aligned}
$$

with

$$
\begin{gathered}
\varepsilon=\frac{1}{2} \sqrt{-2 g^{2}+2 \sqrt{g^{4}+4 \overline{r^{4}}},} \\
\delta=\frac{1}{2} \sqrt{2 g^{2}+2 \sqrt{g^{4}+4 \overline{r^{4}}}}, \\
g^{2}=\overline{g_{m}^{2}}+\bar{s}^{2}, \\
\overline{g_{m}^{2}}=-\left(\frac{e}{b_{m}}+\frac{c}{a}\right) \lambda^{2}, \\
\bar{s}^{2}=\frac{1}{b_{m}}\left(a_{m}-a\right), \\
\overline{r^{4}}=-c \lambda^{2}\left(\frac{a+e \lambda^{2}}{a b_{m}}\right) .
\end{gathered}
$$

Here

$$
\begin{aligned}
a & =\kappa G A, \\
c & =\rho A, \\
e & =\rho I, \\
a_{m} & =\overline{\kappa G A}=\kappa G A-\left(\tau_{u}+\tau_{b}\right) b, \\
b_{m} & =\overline{E I}=\left(E I+2 b h^{2} E_{s}\right) .
\end{aligned}
$$

From (62), the natural frequencies $\lambda=i \omega$ will satisfy

$$
\begin{aligned}
\Delta(i \omega) & \\
= & -\omega^{4} c e\left(d^{\prime}(L)\right)^{2} \\
& +\omega^{2}\left[a c d(L) d^{\prime \prime}(L)-\left(a e+c b_{m}\right) d^{\prime}(L) d^{\prime \prime \prime}(L)\right] \\
& +\left(a^{2}-a a_{m}\right)\left(d^{\prime \prime}(L)\right)^{2}-a b_{m}\left(d^{\prime \prime \prime}(L)\right)^{2}=0
\end{aligned}
$$

The fundamental matrix response $\mathbf{h}(x)$ is obtained in a similar way to the classical case [32]

$$
\begin{aligned}
& \mathbf{h}(x) \\
& =\left(\begin{array}{cc}
\left(a+e \lambda^{2}\right) d(x)-b_{m} d^{\prime \prime}(x) & -a_{m} d^{\prime}(x) \\
a d^{\prime}(x) & -a d^{\prime \prime}(x)+c \lambda^{2} d(x)
\end{array}\right) .
\end{aligned}
$$

We observe from Figure 6 that for frequencies not above the critical frequency, the fundamental response $d(x)$ has lower amplitude when considering surface effects, while for higher frequencies the oscillatory behavior of $d(x)$ follows the classical one.

For a microcantilever beam described by the Timoshenko model with surface effects, the size dependence in the natural frequency of Timoshenko classical model and Timoshenko model including surface effects is illustrated in Figure 7. The solutions based on classical Timoshenko beam theory and Timoshenko beam theory including surface effects are denoted by TB and TMB, respectively. The fundamental natural frequencies are normalized to fundamental frequency of cantilever Euler-Bernoulli beam. In this figure are considered the parameters utilized in [14] for the same purposes. The parameters of surface elasticity and residual surface tension can be determined by molecular dynamics simulations or experiments. Residual surface stresses can be either positive or negative, depending on the crystallographic structure [33]. For an anodic alumina $\mathrm{Al}$ (Young's modulus $E=70 \mathrm{GPa}$, Poisson's ratio $\nu=0.3$ and $\rho=2700 \mathrm{~kg} / \mathrm{m}^{3}$ ) are considered two types of crystallographic direction

$$
\begin{aligned}
A l[100]: E_{s} & =-7.9253 \mathrm{~N} / \mathrm{m} \text { and } \\
\tau & =0.5689 \mathrm{~N} / \mathrm{m}, \\
A l[111]: E_{s} & =5.1882 \mathrm{~N} / \mathrm{m} \text { and } \\
\tau & =0.9108 \mathrm{~N} / \mathrm{m} .
\end{aligned}
$$

In Figure 7, the size dependence effects in the nondimensional natural frequency of TMB microbeams in comparison to the classical TB are illustrated. We can observe that for beam length on the order of nanometer to microns, the difference between natural frequencies is apparent and, by increasing the length of the microbeam, the results tend to Timoshenko classical theory; that is, the surface effects are significant only in nanoscale. This same behavior was observed in [14] for a microbeam simply supported.

\section{Timoshenko Model with Surface Energy in Thick Nanobeams}

In [13], the surface effects are included in the Timoshenko beam model following Gurtin-Murdoch continuum theory [34], in which is considered an elastic surface with zero thickness fully bonded to its bulk material. This elastic surface adds a set of specific constitutive equations relative to distinct material properties of the surface and surface energy effects. Let us denote an isotropic material beam, with rectangular transversal section, length $L$, width $b$, thickness 

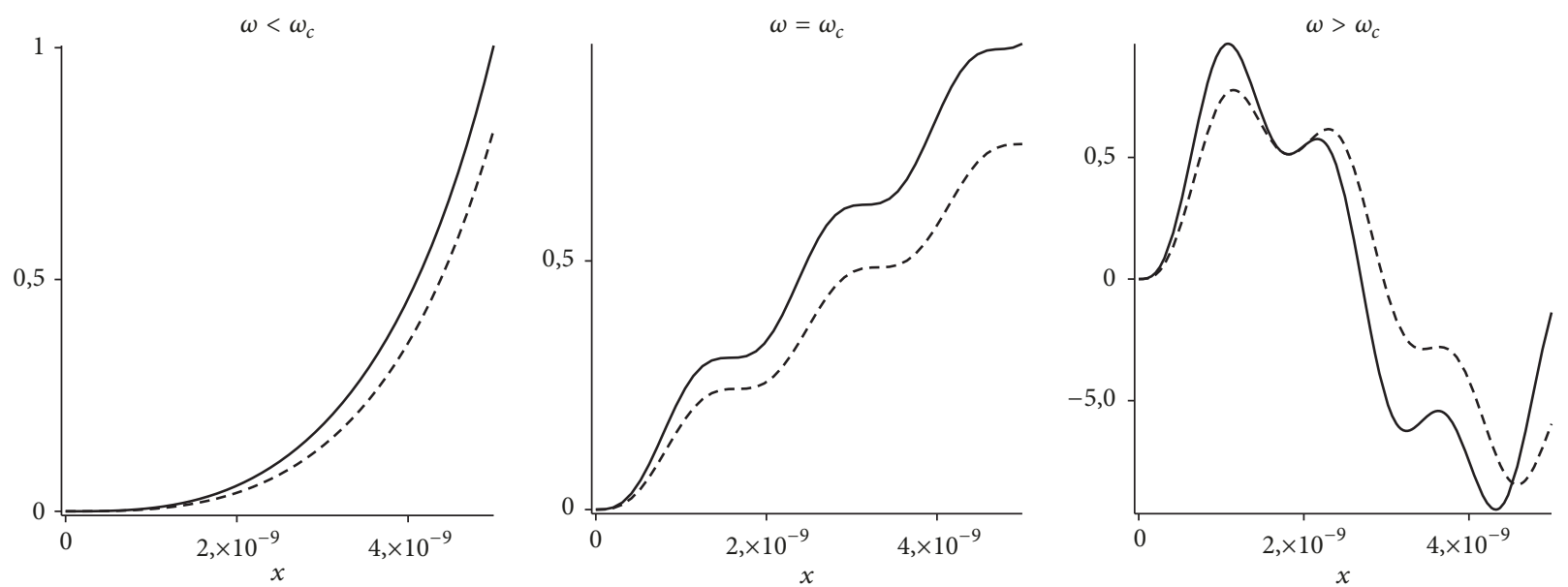

Figure 6: Behavior of the fundamental solution $d(x)$, according to critical natural frequency $\omega_{c}=\sqrt{a / e}$, using $L=5 \times 10^{-9}$ and $A l[111]$ beam configuration, including surface effects (dashed line) and classical case (solid line).

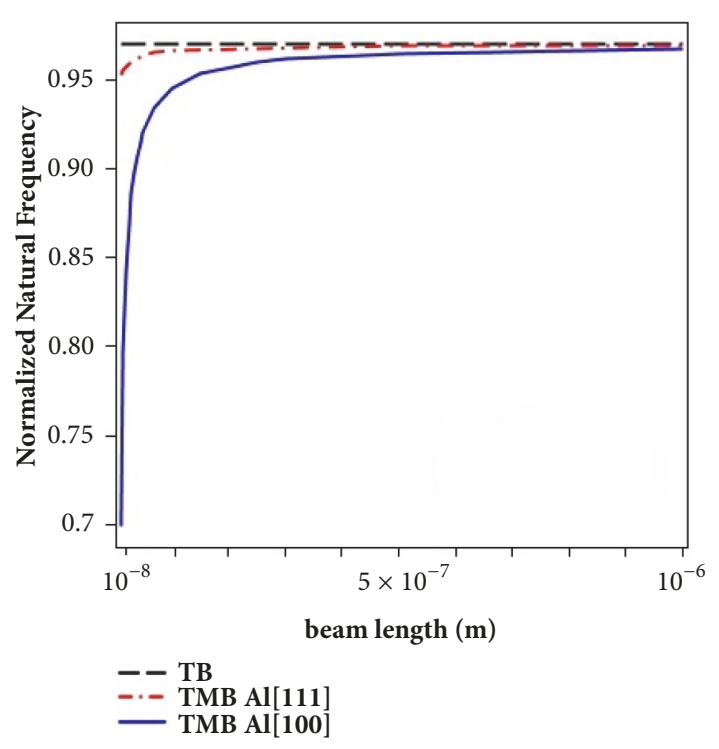

FIGURE 7: Influence of surface effects and size dependence on the normalized fundamental natural frequency of the microcantilever for $2 \mathrm{~h}=0.2 \mathrm{~L}, \mathrm{~b}=0.4 \mathrm{~L}$, and $\kappa=5 / 6$.

$H=2 h$, moment of inertia $I=2 b h^{3} / 3$, and $A=2 b h$ which is the transversal sectional area. The equations for the bulk are the same constitutive equations of classical theory of elasticity. The government equations of transverse and angular displacement, $w(t, x)$ and $\phi(t, x)$, respectively, are then given by

$$
\begin{aligned}
& \left(\rho A+\rho_{0} s^{*}\right) w_{t t}-\left(\kappa G A+\tau_{0} s^{*}\right) w_{x x}+\kappa G A \phi_{x}=0, \\
& \left(\rho I+\rho_{0} I^{*}\right) \phi_{t t}-\left(E I+\left(2 \mu_{0}+\lambda_{0}\right) I^{*}\right) \phi_{x x} \\
& \quad-\frac{2 \nu I \rho_{0}}{H} w_{x t t}+\frac{2 \nu I \tau_{0}}{H} w_{x x x}-\kappa G A\left(w_{x}-\phi\right)=0 .
\end{aligned}
$$

In a compact notation we have

$$
\mathbf{M v}+\mathbf{K v}=0 \text {, }
$$

where

$$
\begin{aligned}
\mathbf{v} & =\left(\begin{array}{l}
w \\
\phi
\end{array}\right), \\
\mathbf{M} & =M_{0}+M_{1} \frac{\partial}{\partial x}, \\
M_{0} & =\left(\begin{array}{cc}
c_{m} & 0 \\
0 & e_{m}
\end{array}\right), \\
M_{1} & =\left(\begin{array}{cc}
0 & 0 \\
-I^{0} & 0
\end{array}\right),
\end{aligned}
$$

and $\mathbf{K}$ is a sum of matrix differential operators

$$
\begin{aligned}
\mathbf{K} & =K_{3} \frac{\partial^{3}}{\partial x^{3}}+K_{2} \frac{\partial^{2}}{\partial x^{2}}+K_{1} \frac{\partial}{\partial x}+K_{0}, \\
K_{3} & =\left(\begin{array}{ll}
0 & 0 \\
I^{1} & 0
\end{array}\right), \\
K_{2} & =\left(\begin{array}{cc}
-a_{m} & 0 \\
0 & -b_{m}
\end{array}\right), \\
K_{1} & =\left(\begin{array}{ll}
0 & a \\
-a & 0
\end{array}\right), \\
K_{0} & =\left(\begin{array}{ll}
0 & 0 \\
0 & a
\end{array}\right),
\end{aligned}
$$

with $c_{m}=\rho A+\rho_{0} s^{*}, a_{m}=\kappa G A+\tau_{0} s^{*}, a=\kappa G A, e_{m}=\rho I+$ $\rho_{0} I^{*}, I^{0}=2 \nu I \rho_{0} / H, I^{1}=2 \nu I \tau_{0} / H$.

The material parameters of bulk are $E, \nu$, and $\rho$, which represent Young's modulus, Poisson's ratio, and mass density, respectively. Also, we have beam surface parameters $\lambda_{0}, \mu_{0}, \tau_{0}$, $\rho_{0}$, Lamé constants, surface residual stress, and mass density of surface. $I^{*}$ is the moment of inertia relative to the surface 
$I^{*}=2 b h^{2}+4 h^{3} / 3$, and for rectangular transversal section $s^{*}=2 b . \kappa$ is the shear correction coefficient.

Rewriting (74), we have

$$
\left(\begin{array}{cc}
c_{m} \frac{\partial^{2}}{\partial t^{2}}-a_{m} \frac{\partial^{2}}{\partial x^{2}} & a \frac{\partial}{\partial x} \\
-I^{0} \frac{\partial^{3}}{\partial t^{2} \partial x}+I^{1} \frac{\partial^{3}}{\partial x^{3}}-a \frac{\partial}{\partial x} & e_{m} \frac{\partial^{2}}{\partial t^{2}}-b_{m} \frac{\partial^{2}}{\partial x^{2}}+a
\end{array}\right) \mathrm{v}
$$

$=0$.

Considering exponential solutions in the form $\mathrm{v}=$ $e^{\lambda t+\beta x} \mathbf{w}$, then

$$
\mathbf{L v}=0
$$

where

$$
\mathbf{L}=\left(\begin{array}{cc}
c_{m} \lambda^{2}-a_{m} \beta^{2} & a \beta \\
-I^{0} \lambda^{2} \beta+I^{1} \beta^{3}-a \beta & e_{m} \lambda^{2}-b_{m} \beta^{2}+a
\end{array}\right)
$$

In order to guarantee $\mathbf{w}$ nonzero, we must have $\operatorname{det}(\mathbf{L})=$ 0 . Thus we obtain the characteristic equation

$$
\begin{aligned}
\Delta(\beta, \lambda)= & c_{m} e_{m} \lambda^{4} \\
& +\left[\left(a I^{0}-b_{m} c_{m}-a_{m} e_{m}\right) \beta^{2}+a c_{m}\right] \lambda^{2} \\
& +\left(a^{2}-a a_{m}\right) \beta^{2}+\left(a_{m} b_{m}-a I^{1}\right) \beta^{4}=0 .
\end{aligned}
$$

7.1. Singular Eigenvalue Problem. By assuming exponential solutions $\mathrm{v}(t, x)=e^{\lambda t} \mathbf{w}(x), \mathbf{w}(x)=\left(\begin{array}{c}W(x) \\ \Psi(x)\end{array}\right)$ in (74), it follows the eigenvalue problem

$$
\left(\lambda^{2} \mathbf{M}+\mathbf{K}\right) \mathbf{w}(x)=\mathbf{0},
$$

which can be written as a singular third-order differential matrix equation

$$
\begin{aligned}
& \mathbb{R} \mathbf{w}^{\prime \prime \prime}(x)+\mathbb{M} \mathbf{w}^{\prime \prime}(x)+\mathbb{C}(\lambda) \mathbf{w}^{\prime}(x)+\mathbb{K}(\lambda) \mathbf{w}(x) \\
& \quad=\mathbf{0}
\end{aligned}
$$

with

$$
\begin{aligned}
\mathbb{R} & =\left(\begin{array}{cc}
0 & 0 \\
I^{1} & 0
\end{array}\right), \\
\mathbb{M} & =\left(\begin{array}{cc}
-a_{m} & 0 \\
0 & -b_{m}
\end{array}\right), \\
\mathbb{C}(\lambda) & =\left(\begin{array}{cc}
0 & a \\
-\left(\lambda^{2} I^{0}+a\right) & 0
\end{array}\right), \\
\mathbb{K}(\lambda) & =\left(\begin{array}{ccc}
\lambda^{2} c_{m} & 0 & \\
0 & \lambda^{2} e_{m}+a
\end{array}\right) .
\end{aligned}
$$

We observe that the nature of the matrix $\mathbb{R}$ characterizes the problem (83) as being singular. Following the spectral methodology, we seek exponential solutions $\mathbf{w}(x)=e^{\beta x} \mathrm{v}$, $v \neq 0$, of $(83)$. It follows that

$$
\Gamma(\beta, \lambda) v=\left(\beta^{3} \mathbb{R}+\beta^{2} \mathbb{M}+\beta \mathbb{C}(\lambda)+\mathbb{K}(\lambda)\right) v=\mathbf{0},
$$

where

$\Gamma(\beta, \lambda)$

$$
=\left(\begin{array}{cc}
-\beta^{2} a_{m} c_{m} \lambda^{2} & \beta a \\
\beta^{3} I^{1}-\beta\left(I^{0} \lambda^{2}+a\right) & -\beta^{2} b_{m}+e_{m} \lambda^{2}+a
\end{array}\right) .
$$

For each $\lambda$, non-zero solutions $v$ can be obtained whenever $\beta$ is a root of the characteristic polynomial

$$
P(\beta, \lambda)=\operatorname{det}\left(\beta^{3} \mathbb{R}+\beta^{2} \mathbb{M}+\beta \mathbb{C}(\lambda)+\mathbb{K}(\lambda)\right)
$$

The characteristic equation

$$
\Delta(\beta, \lambda)=P(\beta, \lambda)=\beta^{4}+g^{2}(\lambda) \beta^{2}-r^{4}(\lambda)=0,
$$

with

$$
\begin{aligned}
& g^{2}(\lambda)=\frac{b_{2}}{b_{0}}=\frac{\left(I^{0} a-b_{m} c_{m}-a_{m} e_{m}\right) \lambda^{2}+a^{2}-a a_{m}}{\left(a_{m} b_{m}-a I^{1}\right)}, \\
& r^{4}(\lambda)=-\frac{b_{4}}{b_{0}}=-\frac{\lambda^{2}\left(a c_{m}+c_{m} e_{m} \lambda^{2}\right)}{\left(a_{m} b_{m}-a I^{1}\right)}
\end{aligned}
$$

has the roots $\beta_{1,2}= \pm \varepsilon$ and $\beta_{3,4}= \pm i \delta$, where

$$
\begin{aligned}
& \varepsilon=\sqrt{\frac{-g^{2}(\lambda)+\sqrt{\left(g^{2}(\lambda)\right)^{2}+4 r^{4}(\lambda)}}{2}}, \\
& \delta=\sqrt{\frac{g^{2}(\lambda)+\sqrt{\left(g^{2}(\lambda)\right)^{2}+4 r^{4}(\lambda)}}{2}}
\end{aligned}
$$

The case of simple roots can be characterized from the condition $P^{\prime}=2 \beta^{3}+g^{2}(\lambda), \beta \neq 0$. This excludes the possibility of $\beta=0$ to be a simple root. Moreover, we can determine the eigenvectors satisfying (85). We summarize these results in the following.

Lemma 1. $\beta$ is a simple root of $P(\beta, \lambda)$ if and only if $\beta \neq 0$ and $\beta^{2} \neq-g^{2}(\lambda) / 2$. For simple roots, the eigenvectors satisfying (85) are given by

$$
\mathrm{v}_{j}=\left(\begin{array}{c}
v_{1 j} \\
\left(\frac{\beta_{j}^{2} a_{m}-\lambda^{2} c_{m}}{\beta_{j} a}\right) v_{1 j}
\end{array}\right)
$$

where $v_{1 j}$ is an arbitrary constant. 


$$
\begin{aligned}
\mathbf{w}(x) & =c_{1} e^{\beta_{1} t} \mathrm{v}_{1}+c_{2} e^{\beta_{2} t} \mathrm{v}_{2}+c_{3} e^{\beta_{2} t} \mathrm{v}_{3}+c_{4} e^{\beta_{4} t} \mathrm{v}_{4} \\
& =\left(c_{1}\left(\frac{\varepsilon^{2} a_{m}-\lambda^{2} c_{m}}{a \varepsilon}\right) c_{2}\left(\frac{\varepsilon^{2} a_{m}-\lambda^{2} c_{m}}{-a \varepsilon}\right) \frac{c_{3}}{i}\left(\frac{-\delta^{2} a_{m}-\lambda^{2} c_{m}}{a \delta}\right) \frac{c_{4}}{i}\left(\frac{-\delta^{2} a_{m}-\lambda^{2} c_{m}}{-a \delta}\right)\right)\left(\begin{array}{c}
c^{\varepsilon x} \\
e^{-\varepsilon x} \\
e^{\delta i x} \\
e^{-\delta i x}
\end{array}\right),
\end{aligned}
$$

and can be also presented in the form

$$
\mathbf{w}(x)
$$

$$
=\left(\begin{array}{cccc}
C_{1} & C_{2} & C_{3} & C_{4} \\
C_{2} K_{a} & C_{1} K_{a} & C_{4} K_{b} & -C_{3} K_{b}
\end{array}\right)\left(\begin{array}{c}
\cosh (\varepsilon x) \\
\sinh (\varepsilon x) \\
\cos (\delta x) \\
\sin (\delta x)
\end{array}\right),
$$

where $C_{1}=c_{1}+c_{2}, C_{2}=c_{1}-c_{2}, C_{3}=c_{3}+c_{4}, C_{4}=\left(c_{3}-c_{4}\right) i$ and

$$
\begin{aligned}
& K_{a}=\frac{a_{m} \varepsilon^{2}-\lambda^{2} c_{m}}{a \varepsilon}, \\
& K_{b}=\frac{a_{m} \delta^{2}-\lambda^{2} c_{m}}{a \delta} .
\end{aligned}
$$

The above representation of $\mathbf{w}(\mathbf{x})$ in terms of hyperbolic and trigonometric functions follows by introducing in (92) the basic change

$$
\left(\begin{array}{c}
e^{\varepsilon x} \\
e^{-\varepsilon x} \\
e^{i \delta x} \\
e^{-i \delta x}
\end{array}\right)=\left(\begin{array}{cccc}
1 & 1 & 0 & 0 \\
1 & -1 & 0 & 0 \\
0 & 0 & 1 & i \\
0 & 0 & 1 & -i
\end{array}\right)\left(\begin{array}{c}
\cosh (\varepsilon x) \\
\sinh (\varepsilon x) \\
\cos (\delta x) \\
\sin (\delta x)
\end{array}\right) .
$$

7.1.1. Repeated Roots Analysis. From the given characterization of simple roots, it is clear that the repeated roots can be only $\beta=0$ and $\beta^{2}=-g^{2}(\lambda) / 2$. The conditions $P^{\prime \prime \prime}=12 \beta=0$ and $P^{(i v)}=12$ exclude the possibility of having nonzero third roots. In such a case, $\beta=0$ will be a quadruple root. More precisely, we have the following.

Lemma 2. (1) If $\beta=0$ is a root of $P(\beta, \lambda)$, then it is a double root if $g^{2}(\lambda) \neq 0$, or quadruple if $g^{2}(\lambda)=0$.

(2) If $\beta=\sqrt{-g^{2}(\lambda) / 2}$ and $\beta=-\sqrt{-g^{2}(\lambda) / 2}$ are roots, with $g^{2}(\lambda) \neq 0$, then they will be double roots.

(3) The characteristic polynomial $P(\beta, \lambda)$ does not have triple roots.

From the above lemmas, critical frequencies values will arise for natural frequencies $\lambda=i \omega$. (i) $\beta=0$ is a root of $P(\beta, i \omega)$, if and only if $r^{4}(\omega)=0$. That is, $r^{4}(i \omega)=0$ for $\omega=0$ or $\omega=\omega_{c}$, with $\omega_{c}=\sqrt{a / e m}$.

(ii) $\beta= \pm \sqrt{-g^{2}(\omega) / 2}, g^{2}(\omega) \neq 0$, is a root of $P(\beta, i \omega)$ if and only if $\left(g^{2}(i \omega)\right)^{2}+4 r^{4}(\omega)=0$, with $r^{4}(\omega) \neq 0$. We have $g^{2}(\omega)=0$ when $\omega=\omega_{g}=$ $\sqrt{\left(a^{2}-a a_{m}\right) /\left(b_{m} c_{m}+a_{m} e_{m}-I^{0} a\right)}$.

7.2. Frequency Equations. Frequency equations will arise when applying boundary conditions as given below.

7.2.1. Simply Supported Beam. In this case we have the following boundary conditions, at $x=0$

$$
\begin{aligned}
w(0) & =0, \\
\omega^{2} I^{0} w(0)-b_{m} \phi^{\prime}(0)+I^{1} w^{\prime \prime}(0) & =0,
\end{aligned}
$$

and at $x=L$

$$
\begin{aligned}
w(L) & =0, \\
\omega^{2} I^{0} w(L)-b_{m} \phi^{\prime}(L)+I^{1} w^{\prime \prime}(L) & =0 .
\end{aligned}
$$

Using the first boundary condition at $x=0$, we obtain $C_{2}+$ $C_{4}=0 \Longrightarrow C_{4}=-C_{2}$. From the second boundary condition we have

$$
\begin{array}{r}
I^{1} w^{\prime \prime}(0)+b_{m} \phi^{\prime}(0)=0, \\
-C_{2} I^{1}\left(\delta^{2}+\varepsilon^{2}\right)+C_{2} b_{m}\left(K_{a} \delta+K_{b} \varepsilon\right)=0
\end{array}
$$

replacing $K_{a}$ and $K_{b}$ and using that $a_{m} b_{m}-I^{1} a>0$ [13], $\varepsilon^{2}+$ $\delta^{2} \neq 0$, results in $C_{2}=C_{4}=0$.

From the boundary conditions at $x=L$, written in matrix form

$$
\left(\begin{array}{ccc}
1 & 0 & 0 \\
\omega^{2} I^{0} & I^{1} & -b_{m}
\end{array}\right)\left(\begin{array}{c}
W(L) \\
W^{\prime \prime}(L) \\
\psi^{\prime}(L)
\end{array}\right)=\mathbf{0}
$$

the system $\mathbf{U c}=0$ is obtained, described by

$$
\left(\begin{array}{ccc}
1 & 0 & 0 \\
\omega^{2} I^{0} & I^{1} & -b_{m}
\end{array}\right)\left(\begin{array}{cc}
\sin (\delta L) & \sinh (\varepsilon L) \\
-\sin (\delta L) \delta^{2} & \varepsilon^{2} \sinh (\varepsilon L) \\
-K_{a} \sin (\delta L) \delta & \varepsilon K_{b} \sinh (\varepsilon L)
\end{array}\right)\left(\begin{array}{l}
C_{1} \\
C_{3}
\end{array}\right)
$$


Since $b_{m} a_{m}-I^{1} a>0$ and $\varepsilon^{2}+\delta^{2} \neq 0$, the frequency equation

$$
\begin{aligned}
\Delta(\omega) & =\operatorname{det}(\mathbf{U}) \\
& =\frac{\left(I^{1} a-b_{m} a_{m}\right)\left(\varepsilon^{2}+\delta^{2}\right)}{a} \sinh (\varepsilon L) \sin (\delta L) \\
& =0
\end{aligned}
$$

reduces to

$$
\sinh (\varepsilon L) \sin (\delta L)=0 .
$$

7.2.2. Cantilever Beam. In this case the boundary conditions are given by

$$
\begin{array}{r}
w(0)=0 \\
\psi(0)=0, \\
a_{m} w^{\prime}(L)-a \psi(L)=0 \\
-b_{m} \psi^{\prime}(L)+I^{1} w^{\prime \prime}(L)+I^{0} \omega^{2} w(L)=0 .
\end{array}
$$

Similarly to the previous case, by using the boundary conditions at $x=0$, we obtain

$$
\begin{aligned}
W(x)= & C_{1}\left(\sin (\delta x)-\frac{K_{a}}{K_{b}} \sinh (\varepsilon x)\right) \\
& +C_{2}(\cos (\delta x)-\cosh (\varepsilon x)), \\
\Psi(x)= & C_{1} K_{a}(\cos (\delta x)-\cos (\varepsilon x)) \\
& -C_{2}\left(K_{a} \sin (\delta x)+K_{b} \sinh (\varepsilon x)\right),
\end{aligned}
$$

The boundary conditions in matrix form at $x=L$

$$
\left(\begin{array}{ccccc}
0 & a_{m} & 0 & -a & 0 \\
\omega^{2} I^{0} & 0 & I^{1} & 0 & -b_{m}
\end{array}\right)\left(\begin{array}{c}
W(L) \\
W^{\prime}(L) \\
W^{\prime \prime}(L) \\
\psi(L) \\
\psi^{\prime}(L)
\end{array}\right)\left(\begin{array}{c}
C_{1} \\
C_{2}
\end{array}\right)=0
$$

lead to a system Uc $=0$ from which we obtain the characteristic equation.

In Table 3 and Figure 8, we present numerical results about natural frequencies and mode shapes. Both cases of supported-supported (S-S) and cantilever (C) boundary conditions are considered. The simulation was done with a silicon beam having the following material and surface parameters [13]:

$$
\begin{aligned}
& E=107 G P a, \\
& \nu=0.33, \\
& \rho=2.33 \times 10^{3} \mathrm{~kg} / \mathrm{m}^{3},
\end{aligned}
$$$$
\rho_{0}=3.17 \times 10^{-7} \mathrm{~kg} / \mathrm{m}^{2} \text {, }
$$

$\mu_{0}=-2.7779 \mathrm{~N} / \mathrm{m}$,

$\lambda_{0}=-4.4939 \mathrm{~N} / \mathrm{m}$,

$\tau_{0}=0.6056 \mathrm{~N} / \mathrm{m}$.

\section{Comments}

(i) From Table 3 and Figure 8, it is observed that the results agree with those found in the literature $[13,14]$.

(ii) When the beam length increases, from nano- to microscale, the results tend to the classical results.

(iii) Natural frequencies can increase or decrease compared to classical results, depending on elastic surface constants signs and boundary conditions (cantilever beam is more influenceable than simply supported beam).

(iv) According to Figure 8, the mode shape component $W(x)$ of a simply supported beam with the inclusion of surface effects is the same of a classical beam. Perceptible differences occur for the cantilever beam case, principally for the first one mode shape, and just in the nanoscale.

\section{Multispan Timoshenko Beams}

The same formulation, which was presented in Section 2 for Euler-Bernoulli multispan beams, can be used with the Timoshenko multispan beam model. We replace the scalar components that appear in the block matrices defined after (1), by the $2 \times 2$ matrix components $M_{j}$ and $K_{j}$

$$
\begin{aligned}
& \mathrm{M}_{\mathrm{j}}=\left(\begin{array}{cc}
\rho_{j} A_{j} & 0 \\
0 & \rho_{j} I_{j}
\end{array}\right), \\
& \mathrm{K}_{\mathrm{j}}=\left(\begin{array}{cc}
-\kappa_{j} G_{j} A_{j} \frac{\partial^{2}}{\partial x^{2}} & \kappa_{j} G_{j} A_{j} \frac{\partial}{\partial x} \\
-\kappa_{j} G_{j} A_{j} \frac{\partial}{\partial x} & -E_{j} I_{j} \frac{\partial^{2}}{\partial x^{2}}+\kappa_{j} G_{j} A_{j}
\end{array}\right) .
\end{aligned}
$$

The components $v_{j}$ become $2 \times 1$ vectors, $v_{j}=\left(\begin{array}{c}w_{j}(t, x) \\ \phi_{j}(t, x)\end{array}\right)$, where $w_{j}(t, x)$ is the flexural displacement and $\phi_{j}(t, x)$ is the angular displacement. Similarly, the components of $F_{j}$ are external loads and moments, respectively, $f_{j}$ and $q_{j}$. Then 
TABLE 3: Comparison between natural frequencies of Timoshenko beam models including and not including surface effects, considering nano- and microscale, of a Silicon beam. Nanobeam geometric parameters: $L=50 \times 10^{-9} \mathrm{~m}, H=2 \mathrm{~h}=6 \times 10^{-9} \mathrm{~m}$, and $\mathrm{b}=3 \times 10^{-9} \mathrm{~m}$. Microbeam geometric parameters: $L=50 \times 10^{-6} \mathrm{~m}, H=2 \mathrm{~h}=6 \times 10^{-6} \mathrm{~m}$, and $b=3 \times 10^{-6} \mathrm{~m}$.

\begin{tabular}{|c|c|c|c|c|c|c|c|c|c|}
\hline \multirow{3}{*}{$\mathrm{BC}$} & \multirow{3}{*}{ Model } & \multicolumn{4}{|c|}{ Nano } & \multirow{2}{*}{\multicolumn{4}{|c|}{$\begin{array}{c}\text { Micro } \\
\text { Natural frequencies } \omega_{i}(\mathrm{MHz})\end{array}$}} \\
\hline & & & tural fre & es $\omega_{i}(\mathrm{GH}$ & & & & & \\
\hline & & $\omega_{1}$ & $\omega_{2}$ & $\omega_{3}$ & $\omega_{4}$ & $\omega_{1}$ & $\omega_{2}$ & $\omega_{3}$ & $\omega_{4}$ \\
\hline \multirow{4}{*}{ S-S } & TMB & 7.0808 & 25.063 & 51.327 & 82.922 & 7.1996 & 27.010 & 55.669 & 89.756 \\
\hline & $\mathrm{TB}$ & 7.1998 & 27.012 & 55.673 & 89.763 & 7.1998 & 27.012 & 55.673 & 89.763 \\
\hline & TMB [13] & 7.08 & 25.07 & 51.33 & 82.92 & - & - & - & - \\
\hline & TB [13] & 7.20 & 27.02 & 55.70 & 89.76 & - & - & - & - \\
\hline \multirow{4}{*}{ C } & TMB & 3.0168 & 14.674 & 36.484 & 64.514 & 2.5976 & 15.273 & 39.297 & 69.725 \\
\hline & $\mathrm{TB}$ & 2.597 & 15.273 & 39.300 & 69.730 & 2.5971 & 15.273 & 39.300 & 69.730 \\
\hline & TMB [13] & 3.02 & 14.67 & 36.38 & 64.46 & - & - & - & - \\
\hline & TB [13] & 2.60 & 15.28 & 39.31 & 69.74 & - & - & - & - \\
\hline
\end{tabular}

$$
\begin{gathered}
\mathbf{v}=\left(\begin{array}{c}
w_{1} \\
\phi_{1} \\
w_{2} \\
\phi_{2} \\
\vdots \\
w_{N} \\
\phi_{N}
\end{array}\right), \\
\mathrm{F}=\left(\begin{array}{c}
f_{1} \\
q_{1} \\
f_{2} \\
q_{2} \\
\vdots \\
f_{N} \\
q_{N}
\end{array}\right) .
\end{gathered}
$$

where $n_{j}$ corresponds to devices or interaction of extremities with the external medium, when they occur, and

$$
\mathbf{w}_{j}(t, x)=\left(\begin{array}{c}
w_{j}(t, x) \\
\phi_{j}(t, x) \\
w_{j}^{\prime}(t, x) \\
\phi_{j}^{\prime}(t, x)
\end{array}\right), \quad j=1: N .
$$

General boundary conditions can be written as

$$
\begin{aligned}
\mathbf{B}_{0} \mathbf{w}_{1}(t, 0) & =\mathbf{n}_{0}, \\
\mathbf{B}_{L} \mathbf{w}_{N}(t, L) & =\mathbf{n}_{L},
\end{aligned}
$$

where

$$
\begin{aligned}
\mathbf{B}_{0} & =\left(\begin{array}{llll}
a_{11} & b_{11} & c_{11} & d_{11} \\
a_{12} & b_{12} & c_{12} & d_{12}
\end{array}\right), \\
\mathbf{n}_{0} & =\left(\begin{array}{l}
n_{1} \\
n_{2}
\end{array}\right), \\
\mathbf{B}_{L} & =\left(\begin{array}{llll}
a_{21} & b_{21} & c_{21} & d_{21} \\
a_{22} & b_{22} & c_{22} & d_{22}
\end{array}\right), \\
\mathbf{n}_{L} & =\left(\begin{array}{l}
n_{3} \\
n_{4}
\end{array}\right),
\end{aligned}
$$

The continuity conditions for displacement, gyro, flexural moment, and shear force in the transversal section discontinuity points $x=x_{j}, j=1: N-1$, including intermediate devices can be written as

$$
\mathscr{C}_{1, i} \mathbf{w}_{i}\left(t, x_{i}\right)=\mathscr{C}_{2, i} \mathbf{w}_{i+1}\left(t, x_{i}\right), \quad i=1: N-1,
$$

where

$$
\begin{aligned}
& \mathscr{C}_{1, i}=\left(\begin{array}{llll}
e_{11}^{(i)} & f_{11}^{(i)} & g_{11}^{(i)} & h_{11}^{(i)} \\
e_{21}^{(i)} & f_{21}^{(i)} & g_{21}^{(i)} & h_{21}^{(i)} \\
e_{31}^{(i)} & f_{31}^{(i)} & g_{31}^{(i)} & h_{31}^{(i)} \\
e_{41}^{(i)} & f_{41}^{(i)} & g_{41}^{(i)} & h_{41}^{(i)}
\end{array}\right), \\
& \mathscr{C}_{2, i}=\left(\begin{array}{llll}
e_{12}^{(i)} & f_{12}^{(i)} & g_{12}^{(i)} & h_{12}^{(i)} \\
e_{22}^{(i)} & f_{22}^{(i)} & g_{22}^{(i)} & h_{22}^{(i)} \\
e_{32}^{(i)} & f_{32}^{(i)} & g_{32}^{(i)} & h_{32}^{(i)} \\
e_{42}^{(i)} & f_{42}^{(i)} & g_{42}^{(i)} & h_{42}^{(i)}
\end{array}\right),
\end{aligned}
$$

and the vector $\mathbf{w}_{i}$ defined in (111).

In what follows, we shall consider double-span beams in which the first segment is modeled by the Timoshenko beam model and the second segment can be modeled by a Timoshenko (TB) or an Euler-Bernoulli (EB) model. 

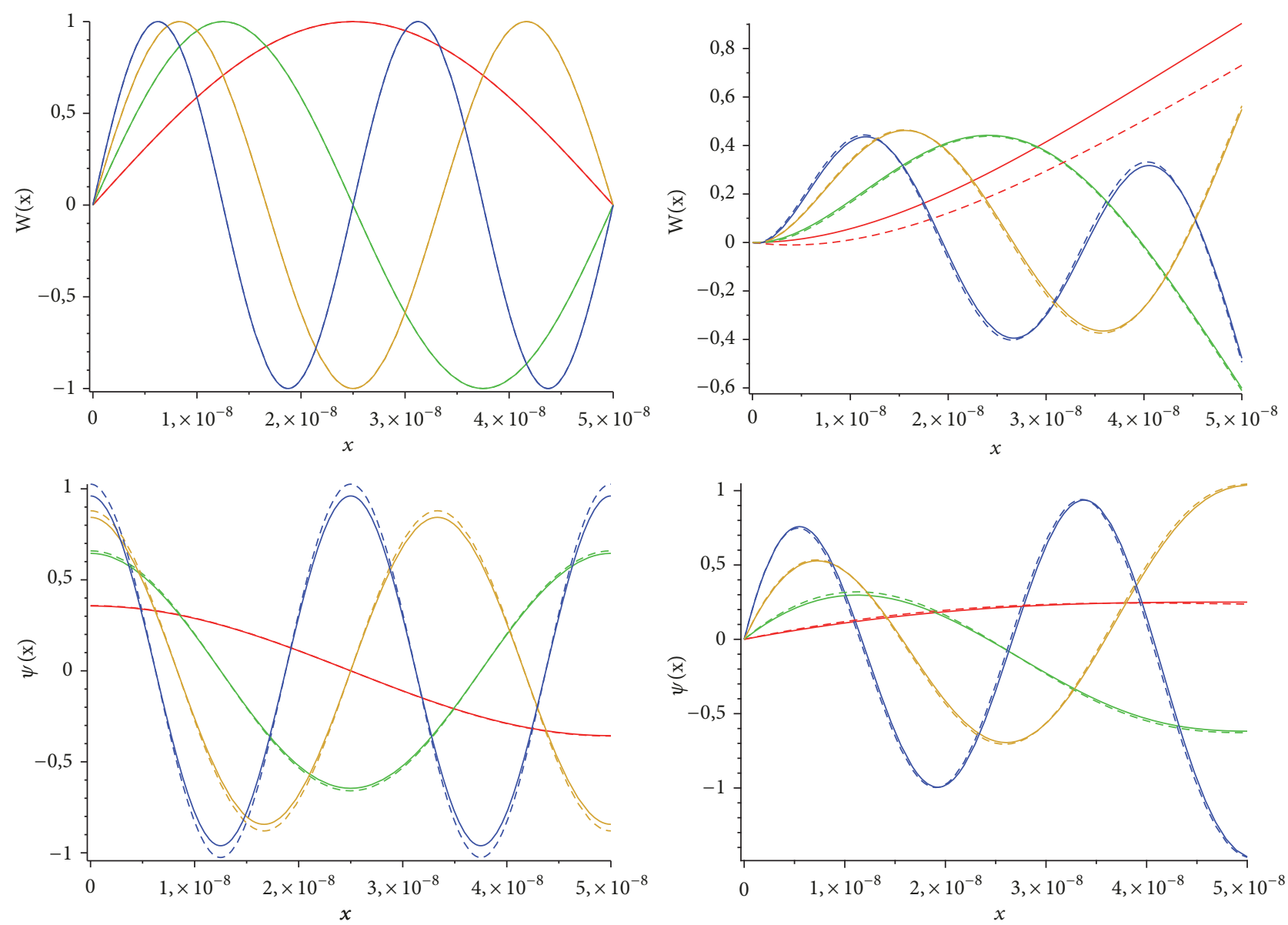

FIGURE 8: Mode shapes of simply supported beam (first column) and cantilever beam (second column), classical case (solid line) and including surface effects (dashed line).

TB-TB. In this case, $w_{j}(t, x)$ and $\phi_{j}(t, x)$ satisfy for $j=1,2$ the equations

$$
\begin{gathered}
\rho_{j} A_{j} \frac{\partial^{2} w_{j}(t, x)}{\partial t^{2}} \\
-\kappa_{j} G_{j} A_{j}\left(\frac{\partial^{2} w_{j}(t, x)}{\partial x^{2}}-\frac{\partial \phi_{j}(t, x)}{\partial x}\right)=0, \\
\rho_{j} I_{j} \frac{\partial^{2} \phi_{j}(t, x)}{\partial t^{2}}-E_{j} I_{j} \frac{\partial^{2} \phi_{j}(t, x)}{\partial x^{2}} \\
-\kappa_{j} G_{j} A_{j}\left(\frac{\partial w_{j}(t, x)}{\partial x}-\phi_{j}(t, x)\right)=0 .
\end{gathered}
$$

For a simply supported beam we have

$$
\begin{gathered}
w_{1}(t, 0)=0, \\
\phi_{1}^{\prime}(t, 0)=0, \\
w_{2}(t, L)=0, \\
\phi_{2}{ }^{\prime}(t, L)=0,
\end{gathered}
$$

and for a cantilever beam

$$
\begin{aligned}
w_{1}(t, 0) & =0, \\
\phi_{1}(t, 0) & =0, \\
\phi_{2}^{\prime}(t, L) & =0, \\
w_{2}^{\prime}(t, L)-\phi_{2}(t, L) & =0 .
\end{aligned}
$$

The compatibility conditions, at the point $x=x_{1}$, without including intermediate devices, are given by

$$
\begin{aligned}
& w_{1}\left(t, x_{1}\right)=w_{2}\left(t, x_{1}\right) \\
& \phi_{1}\left(t, x_{1}\right)=\phi_{2}\left(t, x_{1}\right) \\
& E_{1} I_{1} \phi_{1}^{\prime}\left(t, x_{1}\right)=E_{2} I_{2} \phi_{2}^{\prime}\left(t, x_{1}\right), \\
& \kappa_{1} G_{1} A_{1}\left(w_{1}^{\prime}\left(t, x_{1}\right)-\phi_{1}\left(t, x_{1}\right)\right) \\
& \quad=\kappa_{2} G_{2} A_{2}\left(w_{2}^{\prime}\left(t, x_{1}\right)-\phi_{2}\left(t, x_{1}\right)\right) .
\end{aligned}
$$


The spatial amplitude of a free transversal vibration, at each segment, $\mathrm{v}_{\mathrm{j}}(t, x)=e^{\lambda t} X_{j}(x)$, where $X_{j}(x)=\left(\begin{array}{l}W_{j}(x) \\ \Psi_{j}(x)\end{array}\right)$, for $j=1,2$, satisfies

$$
\left(\lambda^{2} \mathrm{M}_{\mathrm{j}}+\lambda \mathrm{C}_{\mathrm{j}}\right) X_{j}(x)+\mathrm{K}_{\mathrm{j}} X_{j}(x)=0,
$$

or in a differential form

$$
\mathbb{M}_{j} X_{j}^{\prime \prime}+\mathbb{C}_{j} X_{j}^{\prime}+\mathbb{K}_{j}(\lambda) X_{j}=0,
$$

with

$$
\begin{aligned}
\mathbb{M}_{j} & =\left(\begin{array}{cc}
-\kappa_{j} G_{j} A_{j} & 0 \\
0 & -E_{j} I_{j}
\end{array}\right), \\
\mathbb{C}_{j} & =\left(\begin{array}{cc}
0 & \kappa_{j} G_{j} A_{j} \\
-\kappa_{j} G_{j} A_{j} & 0
\end{array}\right), \\
\mathbb{K}_{j} & =\left(\begin{array}{cc}
\lambda^{2} \rho_{j} A_{j} & 0 \\
0 & \rho_{j} I_{j} \lambda^{2}+\kappa G A
\end{array}\right) .
\end{aligned}
$$

The boundary conditions, obtained from (109), can be conveniently written as

$$
\begin{gathered}
\mathbf{B}_{0} \mathbf{X}_{1}(0)=0, \\
\mathbf{B}_{L} \mathbf{X}_{2}(L)=0,
\end{gathered}
$$

and the compatibility conditions, obtained from (112), at $x=$ $x_{1}$

$$
\begin{aligned}
\mathscr{C}_{1,1} \mathbf{X}_{1}\left(x_{1}\right) & =\mathscr{C}_{2,1} \mathbf{X}_{2}\left(x_{1}\right), \\
\mathbf{X}_{j}(x) & =\left(\begin{array}{l}
X_{j}(x) \\
X_{j}^{\prime}(x)
\end{array}\right) .
\end{aligned}
$$

where

$$
\mathbf{X}(x)= \begin{cases}X_{1}(x), & 0 \leq x \leq x_{1} \\ X_{2}(x), & x_{1}<x \leq L .\end{cases}
$$

We choose the fundamental basis at each segment, considering convenient translations,

$$
\begin{aligned}
& \Psi_{1}(x)=\left[\mathbf{h}_{1}(x), \mathbf{h}_{1}^{\prime}(x)\right], \\
& \left.\Psi_{2}(x)=\left[\mathbf{h}_{2}(L-x), \mathbf{h}_{2}^{\prime}(L-x)\right)\right],
\end{aligned}
$$

with $\mathbf{h}_{j}(x)$ being the $2 \times 2$ matrix solution of the initial-value problem

$$
\begin{aligned}
\mathbb{M}_{j} \mathbf{h}_{j}^{\prime \prime}(x)+\mathbb{C}_{j}(\lambda) \mathbf{h}_{j}^{\prime}(x)+\mathbb{K}_{j}(\lambda) \mathbf{h}_{j}(x) & =0, \\
j & =1, \ldots, N . \\
\mathbb{M}_{j} \mathbf{h}_{j}^{\prime}(0) & =I, \\
\mathbf{h}_{j}(0) & =0 .
\end{aligned}
$$

The matrix fundamental solution $\mathbf{h}_{j}$ is given by [25]

$\mathbf{h}_{j}(x)$

$$
=\left(\begin{array}{cc}
a_{j} d_{j}(x)+b_{j} d_{j}^{\prime \prime}(x) & -s_{j} d_{j}(x) \\
s_{j} d_{j}(x) & r_{j} d_{j}(x)-s_{j} d_{j}^{\prime \prime}(x)
\end{array}\right),
$$

where

$$
\begin{aligned}
& a_{j}=\kappa_{j} G_{j} A_{j}+\lambda^{2} \rho_{j} I_{j}, \\
& b_{j}=-E_{j} I_{j}, \\
& r_{j}=\lambda^{2} \rho_{j} A_{j}, \\
& s_{j}=\kappa_{j} G_{j} A_{j} .
\end{aligned}
$$

Then

$$
\begin{aligned}
& X_{1}(x)=\mathbf{h}_{1}(x) \mathbf{c}_{11}+\mathbf{h}_{1}^{\prime}(x) \mathbf{c}_{12}, \\
& X_{2}(x)=\mathbf{h}_{2}\left(x-x_{1}\right) \mathbf{c}_{21}+\mathbf{h}_{2}^{\prime}\left(x-x_{1}\right) \mathbf{c}_{22} .
\end{aligned}
$$

Replacing (128) in the boundary and compatibility conditions we obtained a system $U c=0$, with $U=\mathbf{B} \Phi$,

$$
\mathbf{B}=\left(\begin{array}{cccc}
\mathscr{B}_{0} & 0 & 0 & 0 \\
0 & {\left[\mathscr{C}_{1,1}\right]} & -\left[\mathscr{C}_{2,1}\right] & 0 \\
0 & 0 & \mathbf{0} & \mathscr{B}_{L}
\end{array}\right)_{8 \times 16} .
$$

For a cantilever beam we have

$$
\begin{aligned}
& \mathscr{B}_{0}=\left(\begin{array}{llll}
1 & 0 & 0 & 0 \\
0 & 1 & 0 & 0
\end{array}\right), \\
& \mathscr{B}_{L}=\left(\begin{array}{cccc}
0 & 0 & 0 & 1 \\
0 & -1 & 1 & 0
\end{array}\right) .
\end{aligned}
$$
basis

The matrix $\Phi$ is composed of the elements of the solution

$$
\Phi=\left(\begin{array}{cc}
\Phi_{1}(0) & \mathbf{0} \\
\Phi_{1}\left(l_{1}\right) & \mathbf{0} \\
\mathbf{0} & \Phi_{2}\left(l_{1}\right) \\
\mathbf{0} & \Phi_{2}(L)
\end{array}\right)_{16 \times 8}
$$

where

$$
\begin{aligned}
\Phi_{j}(x) & =\left(\begin{array}{ll}
\mathbf{h}_{j}\left(x-a_{j}\right) & \mathbf{h}_{j}^{\prime}\left(x-a_{j}\right) \\
\mathbf{h}_{j}^{\prime}\left(x-a_{j}\right) & \mathbf{h}_{j}^{\prime \prime}\left(x-a_{j}\right)
\end{array}\right), \quad j=1,2, \\
a_{1} & =0 \\
a_{2} & =L
\end{aligned}
$$

$T B-E B$. We now consider that the movement in the second segment is governed by the Euler-Bernoulli beam model 
(TB-EB). For simplicity, we shall consider Euler-Bernoulli compatibility conditions at the discontinuity point.

In this case, for $0 \leq x \leq x_{1}$ the transversal displacement $w_{T}$ and angular displacement $\phi_{T}$ are governed by the Timoshenko equations

$$
\begin{aligned}
& \rho_{1} A_{1} \frac{\partial^{2} w_{T}(t, x)}{\partial t^{2}} \\
& -\kappa_{1} G_{1} A_{1}\left(\frac{\partial^{2} w_{T}(t, x)}{\partial x^{2}}-\frac{\partial \phi_{T}(t, x)}{\partial x}\right)=0, \\
& \rho_{1} I_{1} \frac{\partial^{2} \phi_{T}(t, x)}{\partial t^{2}}-E_{1} I_{1} \frac{\partial^{2} \phi_{T}(t, x)}{\partial x^{2}} \\
& -\kappa_{1} G_{1} A_{1}\left(\frac{\partial w_{T}(t, x)}{\partial x}-\phi_{T}(t, x)\right)=0,
\end{aligned}
$$

while for $x_{1} \leq x \leq L$, the displacement $w_{E}$ follows the EulerBernoulli model

$$
\rho A_{2} \frac{\partial^{2} w_{E}(t, x)}{\partial t^{2}}+E I_{2} \frac{\partial^{4} w_{E}(x)}{\partial x^{4}}=0 .
$$

The problem can be also described in matrix form as $\mathrm{M} \ddot{v}+$ $\mathrm{K} v=0$, where the block matrix coefficients are

$$
\begin{aligned}
& \mathrm{M}=\left(\begin{array}{cc}
M_{1}^{T} & 0 \\
0 & M_{2}^{E}
\end{array}\right), \\
& \mathrm{K}=\left(\begin{array}{cc}
K_{1}^{T} & 0 \\
0 & K_{2}^{E}
\end{array}\right), \\
& \mathrm{v}=\left(\begin{array}{l}
\mathrm{v}_{1}^{\top} \\
\mathrm{v}_{2}^{\mathrm{E}}
\end{array}\right)=\left(\begin{array}{l}
w_{T}(t, x) \\
\phi_{T}(t, x) \\
w_{E}(t, x)
\end{array}\right),
\end{aligned}
$$

with $M_{1}^{T}, C_{1}^{T}$, and $K_{1}^{T}$ being the $2 \times 2$ matrix coefficients corresponding to Timoshenko equations and $M_{2}^{E}, K_{2}^{E}$ are scalar components corresponding to Euler-Bernoulli equations.

The general boundary conditions are given in $x=0$ according to Timoshenko model, in terms of the state vector $\mathbf{w}_{T}$. And in $x=L$ the general boundary conditions are given as Euler-Bernoulli model; that is,

$$
\begin{aligned}
\mathbf{B}_{0} \mathbf{w}^{T}(t, 0) & =\mathbf{0}, \\
\mathbf{w}_{T}(t, x) & =\left(\begin{array}{c}
w_{T}(t, x) \\
\phi_{T}(t, x) \\
w_{T}^{\prime}(t, x) \\
\phi_{T}^{\prime}(t, x)
\end{array}\right), \\
\mathbf{B}_{L} \mathbf{w}_{E}(t, L) & =\mathbf{0}, \quad \\
\mathbf{w}_{E}(t, x) & =\left(\begin{array}{c}
w_{E}(t, x) \\
w_{E}^{\prime}(t, x) \\
w_{E}^{\prime \prime}(t, x) \\
w_{E}^{\prime \prime \prime}(t, x)
\end{array}\right) .
\end{aligned}
$$

In the discontinuity point $x=x_{1}$, point of section transversal area change, we have chosen compatibility conditions referring to Euler-Bernoulli beam model, having

$$
\begin{aligned}
w_{T}\left(t, x_{1}\right) & =w_{E}\left(t, x_{1}\right), \\
w_{T}^{\prime}\left(t, x_{1}\right) & =w_{E}^{\prime}\left(t, x_{1}\right), \\
E_{1} I_{1} w_{T}^{\prime \prime}\left(t, x_{1}\right) & =E_{2} I_{2} w_{E}^{\prime \prime}\left(t, x_{1}\right), \\
E_{1} I_{1} w_{T}^{\prime \prime \prime}\left(t, x_{1}\right) & =E_{2} I_{2} w_{E}^{\prime \prime \prime}\left(t, x_{1}\right) .
\end{aligned}
$$

The eigensolutions are of the type $\mathbf{v}=\mathrm{e}^{\lambda t} \mathbf{X}(\mathbf{x})$ with

$$
\begin{aligned}
\mathbf{X}(x) & =\left(\begin{array}{c}
X_{1}^{T}(x) \\
X_{2}^{E}(x)
\end{array}\right), \\
X_{1}^{T} & =\left(\begin{array}{l}
W_{T}(x) \\
\Psi_{T}(x)
\end{array}\right), \\
X_{2}^{E} & =W_{E}(x) .
\end{aligned}
$$

The eigenvalue problem $\left(\lambda^{2} \mathrm{M}+\mathrm{K}\right) \mathbf{X}(\mathrm{x})=0$ is equivalent to the differential system

$$
\begin{aligned}
\mathbb{M} X_{T}^{\prime \prime}(x)+\mathbb{C} X_{T}^{\prime}(x)+\mathbb{K}(\lambda) X_{T}(x)=0, & \\
& 0 \leq x \leq x_{1}, \\
X_{E}^{(i v)}(x)-\varepsilon_{2}^{4} X_{E}(x)=0, & \\
& x_{1}<x \leq L,
\end{aligned}
$$

where $\mathbb{M}, \mathbb{C}$, and $\mathbb{K}$ were defined in (120) and $\varepsilon_{2}$ was described in (25).

The boundary conditions can be written as

$$
\begin{aligned}
& \widetilde{B}_{0} \widetilde{\mathbf{X}}_{T}(0)=\mathbf{0}, \\
& B_{L} \widetilde{\mathbf{X}}_{E}(0)=\mathbf{0},
\end{aligned}
$$

where

$$
\begin{gathered}
\widetilde{\mathbf{X}}_{T}(x)=\left(\begin{array}{c}
W_{T}(x) \\
\Psi_{T}(x) \\
W_{T}^{\prime}(x) \\
\Psi_{T}^{\prime}(x) \\
W_{T}^{\prime \prime}(x) \\
W_{T}^{\prime \prime \prime}(x)
\end{array}\right), \\
\widetilde{\mathbf{X}}_{E}=\left(\begin{array}{c}
W_{E}(x) \\
W_{E}^{\prime}(x) \\
W_{E}^{\prime \prime}(x) \\
W_{E}^{\prime \prime \prime}(x)
\end{array}\right) .
\end{gathered}
$$


The compatibility conditions (137) can be written as

$$
\left(\begin{array}{cccccccccc}
1 & 0 & 0 & 0 & 0 & 0 & -1 & 0 & 0 & 0 \\
0 & 0 & 1 & 0 & 0 & 0 & 0 & -1 & 0 & 0 \\
0 & 0 & 0 & 0 & 1 & 0 & 0 & 0 & -\alpha & 0 \\
0 & 0 & 0 & 0 & 0 & 1 & 0 & 0 & 0 & -\alpha
\end{array}\right) \widetilde{\mathbf{X}}\left(x_{1}\right)=\mathbf{0}
$$

with respect to the state span vector $\widetilde{\mathbf{X}}(x)=\left(\begin{array}{c}\widetilde{\mathbf{X}}_{T}(x) \\ \widetilde{\mathbf{X}}_{E}(x)\end{array}\right)$.

The solutions $X_{T}(x)$ and $X_{E}(x)$ can be written using the solutions basis, which were introduced in the previous sections:

$$
\begin{aligned}
X_{T}(x)= & \left(\begin{array}{l}
W_{T}(x) \\
\Psi_{T}(x)
\end{array}\right)=\mathbf{h}(x)\left(\begin{array}{l}
c_{1} \\
c_{2}
\end{array}\right)+\mathbf{h}^{\prime}(x)\left(\begin{array}{c}
c_{3} \\
c_{4}
\end{array}\right), \\
X_{E}(x)= & d(L-x) c_{5}+d^{\prime}(L-x) c_{6}+d^{\prime \prime}(L-x) c_{7} \\
& +d^{\prime \prime \prime}(L-x) c_{8} .
\end{aligned}
$$

By replacing (143) in the boundary conditions and compatibility equations, we obtain a system $\mathbf{U c}=0$, $\mathbf{c}=\left[\begin{array}{llllllll}c_{1} & c_{2} & c_{3} & c_{4} & c_{5} & c_{6} & c_{7} & c_{8}\end{array}\right]^{T}$ and the characteristic equation $\Delta(\lambda)=\operatorname{det}(\mathbf{U})=0$. As before, we $\operatorname{decomp} \mathbf{U}$ as $\mathbf{U}=\mathbf{B} \phi$, where

$$
\boldsymbol{\phi}=\left(\begin{array}{cc}
\Phi_{1}(0) & 0 \\
\Phi_{1}\left(x_{1}\right) & 0 \\
0 & \Phi_{2}\left(x_{1}\right) \\
0 & \Phi_{2}(L)
\end{array}\right)_{20 \times 8}
$$

with

$$
\begin{aligned}
& \phi_{1}=\left(\begin{array}{cc}
h(x)_{2 \times 2} & h^{\prime}(x)_{2 \times 2} \\
h^{\prime}(x)_{2 \times 2} & h^{\prime \prime}(x)_{2 \times 2} \\
\widetilde{h}(x)_{2 \times 2} & \widetilde{h}^{\prime}(x)_{2 \times 2}
\end{array}\right)_{6 \times 4}, \\
& \tilde{h}(x)=\left(\begin{array}{ll}
h^{\prime \prime}(x)[1,1] & h^{\prime \prime}(x)[1,2] \\
h^{\prime \prime \prime}(x)[1,1] & h^{\prime \prime \prime}(x)[1,2]
\end{array}\right), \\
& \phi_{2} \\
& =\left(\begin{array}{llll}
d(L-x) & d^{\prime}(L-x) & d^{\prime \prime}(L-x) & d_{2}^{\prime \prime \prime}(L-x) \\
d^{\prime}(L-x) & d^{\prime \prime}(L-x) & d^{\prime \prime \prime}(L-x) & d^{\prime \prime \prime \prime}(L-x) \\
d^{\prime \prime}(L-x) & d^{\prime \prime \prime}(L-x) & d^{(i v)}(L-x) & d^{(v)}(L-x) \\
d^{\prime \prime \prime}(L-x) & d^{i v}(L-x) & d^{(v)}(L-x) & d_{2}^{(v i)}(L-x)
\end{array}\right)_{4 \times 4}, \\
& \mathbf{B}=\left(\begin{array}{ccc}
{\left[\widetilde{B}_{0}\right]_{2 \times 6}} & 0 & 0 \\
0 & {\left[B_{x_{1}}\right]_{4 \times 10}} & 0 \\
0 & 0 & {\left[B_{L}\right]_{2 \times 4}}
\end{array}\right) .
\end{aligned}
$$

8.1. Simulations. The two span beam models considered have been compared with results of Euler-Bernoulli and Timoshenko double-span beam models in dimensional and unidimensional formulations.
TABLE 4: Parameters of the double-span beam [29].

\begin{tabular}{lcc}
\hline Parameters & Numeric value & Unity \\
\hline Length $l_{1}$ & 0,254 & $\mathrm{~m}$ \\
Length $l_{2}$ & 0,140 & $\mathrm{~m}$ \\
Width $w$ & 0,02545 & $\mathrm{~m}$ \\
Thickness $t_{1}$ & 0,01905 & $\mathrm{~m}$ \\
Thickness $t_{2}$ & 0,00549 & $\mathrm{~m}$ \\
Mass density $\rho$ & 2830 & $\mathrm{Kg} / \mathrm{m}^{3}$ \\
Young's modulus $E$ & 71,7 & $\mathrm{GPa}$ \\
Moment of inertia $I_{1}$ & $1,4633 \times 10^{-8}$ & $\mathrm{~m}^{4}$ \\
Moment of inertia $I_{2}$ & $3,502 \times 10^{-10}$ & $\mathrm{~m}^{4}$ \\
\hline
\end{tabular}

Dimensional Model. We assume a double-span beam, with transversal section discontinuity due to variable thickness. This beam has constant width, but different thickness. The geometric and materials properties of this beam are presented in Table 4.

We present results about natural frequencies, of three cases: two Euler-Bernoulli segments (EB-EB), two Timoshenko segments (TB-TB), and coupled case (TB-EB), in Table 5.

We observe from Table 5 that the values of natural frequencies of the coupled beam (TB-EB) are between the respective values obtained by $\mathrm{TB}-\mathrm{TB}$ and $\mathrm{EB}-\mathrm{EB}$ cases, wherein the EB-EB natural frequencies, as expected, are greater than the others, in all boundary conditions cases presented: S-S (simply supported), C-F (clamped free), and F-F (free-free). Moreover, in the case of free-free boundary condition, as expected, TB-TB produces the most similar results to those obtained experimentally in [29].

Dimensionless Model. We now consider the two span models with classical dimensionless variables. For the EulerBernoulli beam model, the spatial problem in a dimensionless form is given by

$$
\frac{d^{4} \bar{X}_{j}\left(X_{j}\right)}{d X_{j}}-\Omega_{j}^{4} \bar{X}_{j}\left(X_{j}\right)=0,
$$

where the dimensionless parameters are $X_{j}=$ $x_{j} / L, \bar{X}_{j}\left(X_{j}\right)=X_{j} / L, \Omega_{j}^{4}=\rho_{j} A_{j} \omega^{2} / E_{j} I_{j}, \Omega_{j}^{4}=\mu_{j} \Omega_{1}^{4}$.

The Timoshenko model in a dimensionless matrix form is given by

$$
\begin{gathered}
{\left[\begin{array}{ll}
\xi_{j} & 0 \\
0 & 1
\end{array}\right]\left[\begin{array}{l}
Y_{j}^{\prime \prime}(X) \\
\Psi_{j}^{\prime \prime}(X)
\end{array}\right]+\left[\begin{array}{cc}
0 & -\xi_{j} \\
\xi_{j} & 1
\end{array}\right]\left[\begin{array}{l}
Y_{j}^{\prime}(X) \\
\Psi_{j}^{\prime}(X)
\end{array}\right]} \\
+\left[\begin{array}{cc}
\Omega_{j}^{2} & 0 \\
0 & -\xi_{j}+\eta_{j} \Omega_{j}^{2}
\end{array}\right]\left[\begin{array}{l}
Y_{j}(X) \\
\Psi_{j}(X)
\end{array}\right]=\left[\begin{array}{l}
0 \\
0
\end{array}\right],
\end{gathered}
$$


TABLE 5: Comparison of natural frequencies from the double-span beam. EB-EB: two segments of Euler-Bernoulli beam, TB-TB: two segments of Timoshenko beam, and TB-EB: first segment using Euler-Bernoulli beam model and second segment using Timoshenko beam model.

\begin{tabular}{lccccc}
\hline Case & Model & \multicolumn{3}{c}{ Natural frequencies $\omega_{i}(\mathrm{~Hz})$} & \multicolumn{2}{c}{$\omega_{3}$} & $\omega_{2}$ \\
& & $\omega_{1}$ & 782.09 & 1300.56 & 2874.69 \\
S-S & EB-EB & 101.77 & 777.85 & 1291.65 & 2816.98 \\
& TB-EB & 101.74 & 775.85 & 1285.65 & 2796.89 \\
\hline \multirow{3}{*}{ C-F } & TB-TB & 101.64 & 326.69 & 1170.27 & 1154.90 \\
& EB-EB & 139.56 & 325.84 & 1150.82 & 1797.41 \\
& TB-EB & 139.39 & 325.45 & 1804.01 & 1761.78 \\
& TB-TB & 139.31 & 1181.28 & 1775.8 & 3603.04 \\
F-F & EB-EB & 292.42 & 1775.94 & 1786.63 & 3512.95 \\
& TB-EB & 292.14 & 1167.89 & $1759-1771$ \\
\hline
\end{tabular}

TABLE 6: The effects of the change of discontinuity point $R_{1}=x_{1} / L$ in the dimensionless natural frequencies for a cantilever double span beam with variations of thickness.

\begin{tabular}{|c|c|c|c|c|c|c|c|c|c|}
\hline \multirow{3}{*}{ Case } & \multirow{3}{*}{ Model } & \multicolumn{5}{|c|}{ Configuration A } & \multicolumn{2}{|c|}{ Configuration B } & \multirow[b]{3}{*}{$\Omega^{[4]}$} \\
\hline & & \multicolumn{7}{|c|}{ Dimensionless natural frequencies $\Omega^{[i]}$} & \\
\hline & & $\Omega^{[1]}$ & $\Omega^{[2]}$ & $\Omega^{[3]}$ & $\Omega^{[4]}$ & $\Omega^{[1]}$ & $\Omega^{[2]}$ & $\Omega^{[3]}$ & \\
\hline \multirow{3}{*}{$R_{1}=0.2$} & TB-TB & 1.4093 & 3.4619 & 5.6243 & 7.4545 & 1.7184 & 4.0974 & 6.5148 & 8.7633 \\
\hline & TB-EB & 1.4098 & 3.4721 & 5.6637 & 7.5438 & 1.7193 & 4.1198 & 6.5990 & 8.9637 \\
\hline & EB-EB & 1.4101 & 3.4769 & 5.6854 & 7.6159 & 1.7209 & 4.1381 & 6.6554 & 9.077 \\
\hline \multirow{3}{*}{$R_{1}=0.5$} & TB-TB & 1.9972 & 3.5642 & 5.9015 & 8.2377 & 2.0150 & 4.0913 & 6.8545 & 9.1581 \\
\hline & TB-EB & 1.9985 & 3.5725 & 5.9372 & 8.3063 & 2.0162 & 4.1061 & 6.9121 & 9.3086 \\
\hline & EB-EB & 2.0004 & 3.5901 & 5.9831 & 8.5546 & 2.0198 & 4.1331 & 7.050 & 9.5862 \\
\hline \multirow{3}{*}{$R_{2}=0.8$} & TB-TB & 2.0982 & 4.7587 & 6.6143 & 9.1855 & 1.9918 & 4.7325 & 7.3808 & 9.7778 \\
\hline & TB-EB & 2.1000 & 4.7673 & 6.6436 & 9.2371 & 1.9933 & 4.7395 & 7.4083 & 9.8619 \\
\hline & EB-EB & 2.1046 & 4.8267 & 6.7614 & 9.6670 & 1.9973 & 4.8113 & 7.6241 & 10.329 \\
\hline
\end{tabular}

with the dimensionless parameters defined by

$$
\begin{aligned}
X & =\frac{x}{L} ; \\
Y_{j}(X) & =\frac{Y_{j}(x)}{L} ; \\
\Psi_{j}(X) & =\psi_{j}(x) ; \\
\Omega_{j}^{2} & =\frac{(\rho A)_{j} \omega^{2} l^{4}}{(E I)_{j}} ; \\
\eta_{j} & =\frac{I_{j}}{A_{j} L^{2}} ; \\
\xi_{j} & =\frac{\kappa G_{j} A_{j} L^{2}}{(E I)_{j}}=\frac{\kappa}{2 \eta_{j}\left(1+v_{j}\right)} .
\end{aligned}
$$

Then we have the dimensionless frequencies by each segment

$$
\begin{aligned}
& \Omega_{j}^{2}=\frac{(\rho A)_{j} \omega^{2} L^{4}}{(E I)_{j}} \quad(\text { Timoshenko }), \\
& \Omega_{j}^{4}=\frac{(\rho A)_{j} \omega^{2} L^{4}}{(E I)_{j}} \quad(\text { Euler }- \text { Bernoulli }) .
\end{aligned}
$$

These frequencies can be written as a relation of $\Omega_{1} \cdot \Omega_{j}=\left(\mu_{j}\right)^{1 / 2} \Omega_{1}$, for Timoshenko beam, and $\Omega_{j}=\left(\mu_{j}\right)^{1 / 4} \Omega_{1}$ for Euler-Bernoulli beam, being $\mu_{j}=\left((\rho A)_{j} /(\rho A)_{1}\right)\left((E I)_{1} /(E I)_{j}\right)$.

In Table 6 we present results about dimensionless natural frequencies; we have considered variations in the position of the discontinuity point $R_{j}=x_{j} / L$ and variations of thickness of the second segment; constant width is considered, according to the following configurations.

Configuration A: double-span beam with variable thickness and constant width, $t_{1}=0.08, t_{2}=0.03, w_{1}=w_{2}=0.03$.

Configuration B: double-span beam with variable thickness and constant width, $t_{1}=0.08, t_{2}=0.05, w_{1}=w_{2}=0.03$.

Comparative results of dimensionless natural frequencies are presented in Table 6. 
We observe from Table 6 that the values of dimensionless natural frequencies of the coupled beam (TB-EB) are between the respective values obtained by TB-TB and EB-EB cases, wherein the EB-EB natural frequencies, as expected, are greater than the others. Also, we can observe that when the thickness of the second segment increases, the natural frequencies become greater, mainly the higher frequencies (third and fourth frequencies), except for the case $R_{1}=0.8$ in which the first and second frequencies become greater. When $R_{1}$ goes from 0.2 to 0.5 , all frequencies increase, but when $R_{1}$ goes from 0.5 to 0.8 , this occurs only for Configuration $\mathrm{A}$, and for Configuration $\mathrm{B}$ the first one frequency does not increase in this case.

\section{Conclusions}

This paper formulated in matrix form the time domain determination of forced responses and in the frequency domain the obtention of modes of elastic models that can be used in AFM when subjected to tip-sample interactions.

Forced responses are determined by convolution of the input load with the time domain impulse matrix function. The corresponding matrix transfer function and modes of a multispan cantilever beam were determined in terms of solution basis which have the same shape and are generated by a fundamental solution.

It was observed that the spatial amplitude diminishes with the inclusion of a piezoelectric patch when the pulse is positioned in the piezoelectric layer segment; this effect does not appear when the pulse is positioned in the third segment. The reduction of three segments for two segments, maintaining the others material and geometric properties, influences the magnitude of the natural frequencies. When the piezoelectric patch is removed, then the frequencies decrease.

We worked with two Timoshenko beam models including surface effects through different approaches and changing the classical Timoshenko equations in different manners. For both models were presented the results which evidence that, in agreement with the literature $[13,14]$, for beam length on the order of nanometer to microns, the difference between natural frequencies is apparent and, by increasing the length of the microbeam, the results tend to Timoshenko classical theory; that is, the surface effects are significant only in nanoscale.

Simulations were performed with double-span beams in which the first segment is modeled by the Timoshenko beam model and the second segment can be modeled by a Timoshenko (TB) or an Euler-Bernoulli (EB) model. Consistent results of TB-EB beams were obtained, in dimensional and dimensionless forms, when compared with EB-EB and TB$\mathrm{TB}$ cases. The value of frequencies for this model (TB-EB) is located between the natural frequencies of uniform doublespan models TB-TB and EB-EB.

\section{Data Availability}

The data used to support the findings of this study are included within the article.

\section{Conflicts of Interest}

We have no conflicts of interest.

\section{Acknowledgments}

This work was performed as part of the employment of the authors at their institutions and the third author held a fellowship no. 140823/2014-0 from CNPq of Brazil.

\section{References}

[1] B. Voigtlander, Scanning Probe Microscopy:Atomic Force Microscopy and Scanning Tunneling Microscopy, Springer, 2016.

[2] V. M. Harik, "Mechanics of carbon nanotubes: applicability of the continuum-beam models," Computational Materials Science, vol. 24, no. 3, pp. 328-342, 2002.

[3] E. B. Magrab, Vibrations of elastic systems: with applications to MEMS and NEMS, vol. 184, Springer Science and Business Media, 2012.

[4] F. Ebrahimi and M. R. Barati, "A modified nonlocal couple stress-based beam model for vibration analysis of higherorder FG nanobeams," Mechanics of Advanced Materials and Structures, pp. 1-12, 2017.

[5] M. K. Moutlana and S. Adali, "Fundamental frequencies of a nano beam used for atomic force microscopy (AFM) in tapping mode," MRS Advances, vol. 3, no. 42-43, pp. 2617-2626, 2018.

[6] R. Gholami, R. Ansari, and Y. Gholami, "Size-dependent bending, buckling and vibration of higher-order shear deformable magneto-electro-thermo-elastic rectangular nanoplates," Materials Research Express, vol. 4, no. 6, 2017.

[7] A. M. Dehrouyeh-Semnani and A. Bahrami, "On sizedependent Timoshenko beam element based on modified couple stress theory," International Journal of Engineering Science, vol. 107, pp. 134-148, 2016.

[8] N. Togun and S. M. Bağdatli, "Size dependent nonlinear vibration of the tensioned nanobeam based on the modified couple stress theory," Composites Part B: Engineering, vol. 97, pp. 255-262, 2016.

[9] A. Salehi-Khojin, S. Bashash, and N. Jalili, "Modeling and experimental vibration analysis of nanomechanical cantilever active probes," Journal of Micromechanics and Microengineering, vol. 18, no. 8, 2008.

[10] B. Farshi, A. Assadi, and A. Alinia-Ziazi, "Frequency analysis of nanotubes with consideration of surface effects," Applied Physics Letters, vol. 96, no. 9, 2010.

[11] R. E. Miller and V. B. Shenoy, "Size-dependent elastic properties of nanosized structural elements," Nanotechnology, vol. 11, no. 3, pp. 139-147, 2000.

[12] H. S. Zhao, Y. Zhang, and S. T. Lie, "Frequency equations of nonlocal elastic micro/nanobeams with the consideration of the surface effects," Applied Mathematics and Mechanics-English Edition, vol. 39, no. 8, pp. 1089-1102, 2018.

[13] C. Liu and R. K. N. D. Rajapakse, "Continuum models incorporating surface energy for static and dynamic response of nanoscale beams," IEEE Transactions on Nanotechnology, vol. 9, no. 4, pp. 422-431, 2010.

[14] S. Abbasion, A. Rafsanjani, R. Avazmohammadi, and A. Farshidianfar, "Free vibration of microscaled Timoshenko beams," Applied Physics Letters, vol. 95, no. 14, 2009. 
[15] R. Ghaffari and R. A. Sauer, "Modal analysis of graphenebased structures for large deformations, contact and material nonlinearities," Journal of Sound and Vibration, vol. 423, pp. 161179, 2018.

[16] Z. Guo, M. Sheng, and T. Wang, "Flexural wave attenuation in a periodic laminated beam," American Journal of Engineering Research (AJER), vol. 5, no. 6, pp. 258-265, 2016.

[17] C. Abels, V. Mastronardi, F. Guido et al., "Nitride-Based Materials for Flexible MEMS Tactile and Flow Sensors in Robotics," Sensors, vol. 17, no. 5, p. 1080, 2017.

[18] F. L. Forgerini and R. Marchiori, "A brief review of mathematical models of thin film growth and surfaces," Biomatter, vol. 4, no. 1, 2014.

[19] M. Shaat, "Mode localization phenomenon in microbeams due to surface roughness," https://arxiv.org/abs/1803.02638, pp. 218, 2018.

[20] M. Marrese, V. Guarino, and L. Ambrosio, "Atomic Force Microscopy: A Powerful Tool to Address Scaffold Design in Tissue Engineering," Journal of Functional Biomaterials, vol. 8, no. 1, p. 7, 2017.

[21] Y. F. Dufrêne, T. Ando, R. Garcia et al., "Imaging modes of atomic force microscopy for application in molecular and cell biology," Nature Nanotechnology, vol. 12, no. 4, pp. 295-307, 2017.

[22] W. Chang, H. Lee, and Y. Yang, "Mathematical modeling of nanomachining with atomic force microscope cantilevers," Journal of Physics: Conference Series, vol. 490, article no. 012161, 2014.

[23] A. V. Aksenov, "Method of construction of the Riemann function for a second-order hyperbolic equation," Journal of Physics: Conference Series, vol. 937, article no. 012001, 2017.

[24] M. H. Korayem, S. Zafari, A. Amanati, M. Damircheli, and N. Ebrahimi, "Analysis and control of micro-cantilever in dynamic mode AFM," The International Journal of Advanced Manufacturing Technology, vol. 50, no. 9-12, pp. 979-990, 2010.

[25] J. R. Claeyssen, "The timoshenko beam model in vibrating afm cantilevers," in Proceedings of the 9th Brazilian Conference on Dynamics Control and their Applications, J. M. Balthazar, Ed., pp. 60-70, 2010.

[26] H. K. Hong and J. T. Chen, "On the dual representation and its application to vibration problems," in Boundary Elements $X V$ Stress Analysis, vol. 2, 1988.

[27] J. R. Claeyssen and I. F. Ferreira, A resposta impulso em modelos evolutivos e estacionários, Universidade Federal de Santa Maria, Santa Maria,Brazil, 2009.

[28] S. Kukla and I. Zamojska, "Frequency analysis of axially loaded stepped beams by Green's function method," Journal of Sound and Vibration, vol. 300, no. 3-5, pp. 1034-1041, 2007.

[29] S. C. Stanton and B. P. Mann, "On the dynamic response of beams with multiple geometric or material discontinuities," Mechanical Systems and Signal Processing, vol. 24, no. 5, pp. 1409-1419, 2010.

[30] N. Jalili, Piezoelectric-Based Vibration Control From Macro to Micro/Nano Scale Systems, Springer, 2010.

[31] M. Zhu, W. Zhe, and Z. M. Juan, "Dynamic analysis of timoshenko beam with arbitrary constraints and a further optimization based on least energy principle," Mathematical Problems in Engineering, vol. 2018, Article ID 1269738, 19 pages, 2018.

[32] J. R. Claeyssen and S. N. J. Costa, "Modes for the coupled Timoshenko model with a restrained end," Journal of Sound and Vibration, vol. 296, no. 4-5, pp. 1053-1058, 2006.
[33] G.-F. Wang and X.-Q. Feng, "Effects of surface elasticity and residual surface tension on the natural frequency of microbeams," Applied Physics Letters, vol. 90, no. 23, Article ID 231904, 2007.

[34] M. E. Gurtin and A. Ian Murdoch, "Surface stress in solids," International Journal of Solids and Structures, vol. 14, no. 6, pp. 431-440, 1978. 


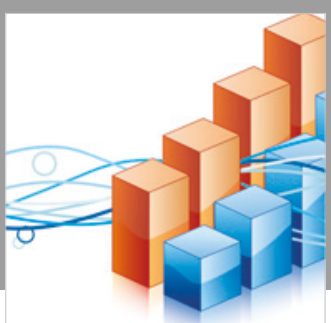

Advances in

Operations Research

\section{-n-m}
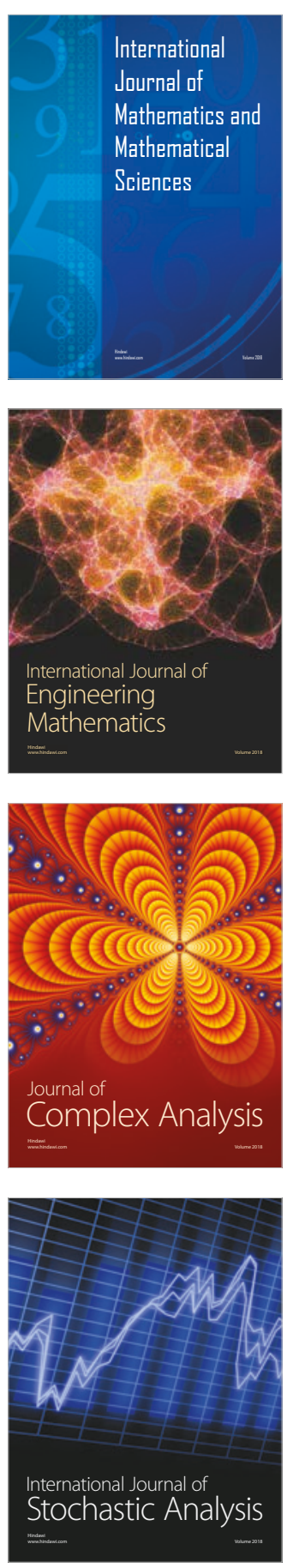
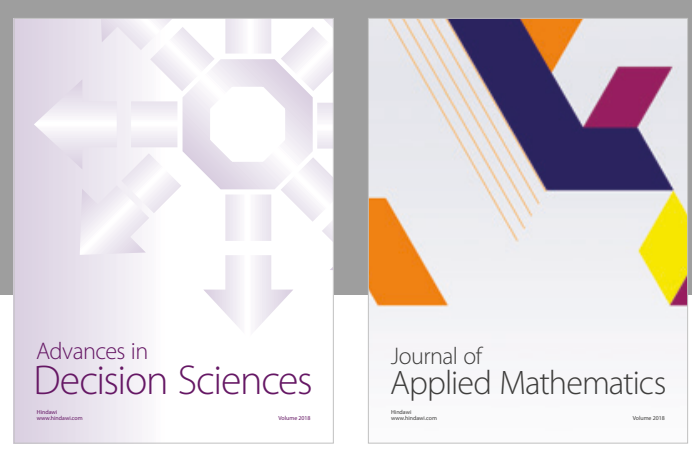

Journal of

Applied Mathematics
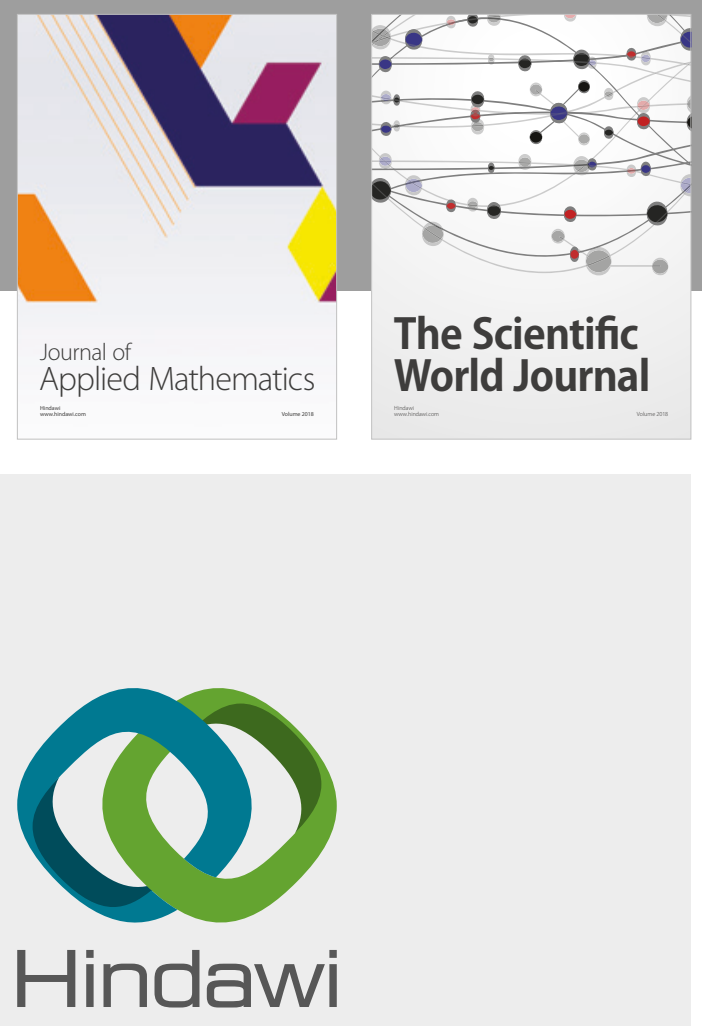

Submit your manuscripts at

www.hindawi.com

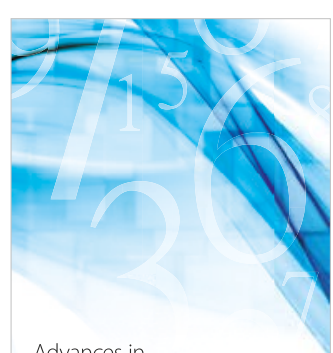

Advances in
Numerical Analysis
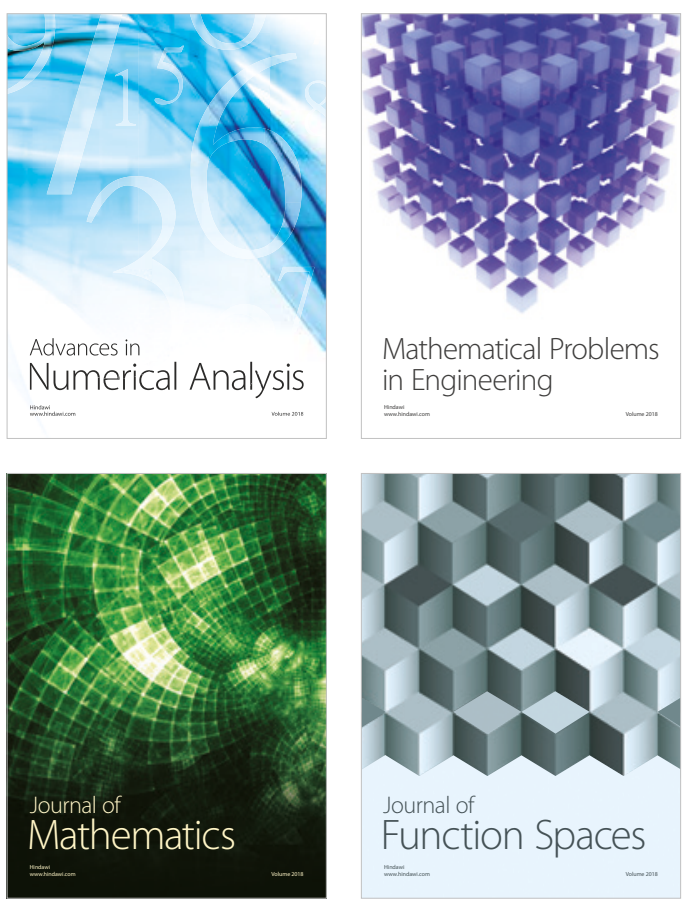

Mathematical Problems in Engineering

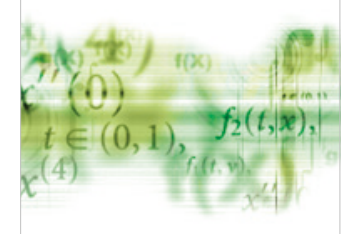

International Journal of

Differential Equations

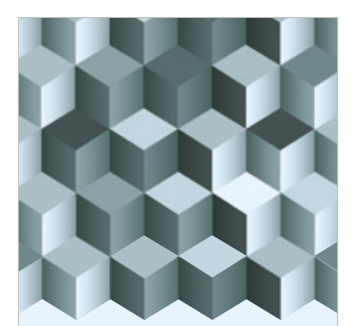

Journal of

Function Spaces
The Scientific

World Journal

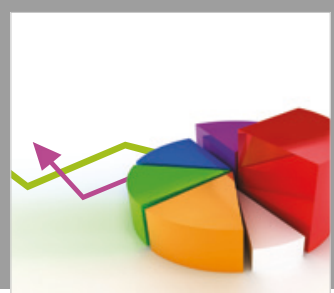

Journal of

Probability and Statistics
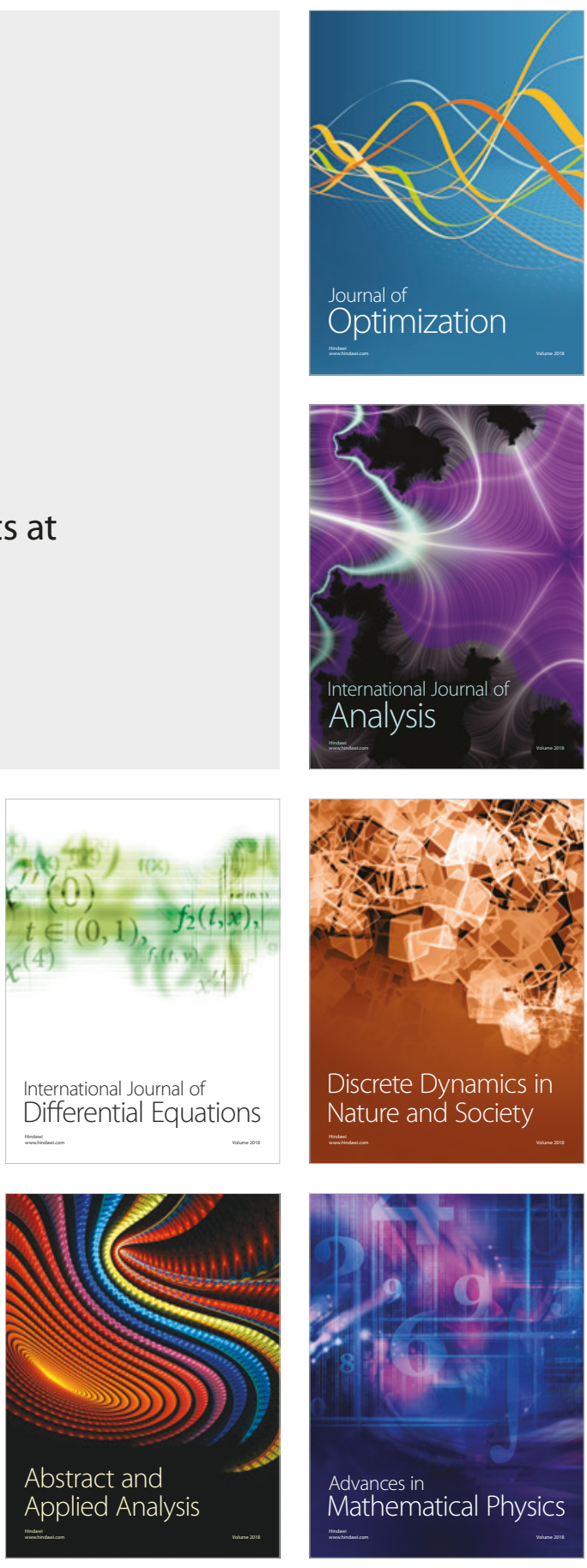